\title{
Integrating gene expression, spatial location and histology to identify spatial domains and spatially variable genes by graph convolutional network
}

Mingyao Li ( $\sim$ mingyao@mail.med.upenn.edu )

University of Pennsylvania https://orcid.org/0000-0003-2422-9494

Jian Hu

University of Pennsylvania

Xiangjie Li

Chinese Academy of Medical Sciences and Peking Union Medical College

Kyle Coleman

University of Pennsylvania

Amelia Schroeder

University of Pennsylvania

David Irwin

University of Pennsylvania

Edward Lee

University of Pennsylvania

Russell Shinohara

University of Pennsylvania

\section{Article}

Keywords: spatial transcriptomics, histology, spatial domains, spatially variable genes, graph convolutional network

Posted Date: December 10th, 2020

DOl: https://doi.org/10.21203/rs.3.rs-119776/v1

License: (c) (i) This work is licensed under a Creative Commons Attribution 4.0 International License. Read Full License

Version of Record: A version of this preprint was published at Nature Methods on October 28th, 2021. See the published version at https://doi.org/10.1038/s41592-021-01255-8. 
1 Integrating gene expression, spatial location and histology to identify spatial

2 domains and spatially variable genes by graph convolutional network

4 Jian $\mathrm{Hu}^{1,}{ }^{,}$, Xiangjie $\mathrm{Li}^{2}$, Kyle Coleman ${ }^{1}$, Amelia Schroeder ${ }^{1}$, David J. Irwin ${ }^{3}$, Edward B. Lee, ${ }^{4,5}$, Russell T.

5 Shinohara ${ }^{1}$, Mingyao $\mathrm{Li}^{1,{ }^{*}}$

6

7 1. Department of Biostatistics, Epidemiology and Informatics, Perelman School of Medicine, University of

8 Pennsylvania, Philadelphia, PA 19104, USA.

9 2. State Key Laboratory of Cardiovascular Disease, Fuwai Hospital, National Center for Cardiovascular

10 Diseases, Chinese Academy of Medical Sciences and Peking Union Medical College, Beijing 100037, China.

11 3. Department of Neurology, Perelman School of Medicine, University of Pennsylvania, Philadelphia, PA 12 19104, USA.

13 4. Department of Pathology and Laboratory Medicine, Perelman School of Medicine, University of 14 Pennsylvania, Philadelphia, PA 19104, USA.

15 5. Translational Neuropathology Research Laboratory, Department of Pathology and Laboratory Medicine,

16 Perelman School of Medicine, University of Pennsylvania, Philadelphia, PA 19104, USA.

17

18 Correspondence:

19 Jian Hu, jianhu@pennmedicine.upenn.edu

20 Mingyao Li, $\underline{\text { mingyao@pennmedicine.upenn.edu }}$

22 Key words: spatial transcriptomics; histology; spatial domains; spatially variable genes; graph 23 convolutional network. 


\section{Abstract}

26 Recent advances in spatial transcriptomics technologies have enabled comprehensive characterization of

27 gene expression patterns in the context of tissue microenvironment. To elucidate spatial gene expression

28 variation, we present SpaGCN, a graph convolutional network approach that integrates gene expression,

29 spatial location and histology in spatial transcriptomics data analysis. Through graph convolution, SpaGCN

30 aggregates gene expression of each spot from its neighboring spots, which enables the identification of

31 spatial domains with coherent expression and histology. The subsequent domain guided differential

32 expression analysis then detects genes with enriched expression patterns in the identified domains.

33 Analyzing five spatially resolved transcriptomics datasets using SpaGCN, we show it can detect genes with

34 much more enriched spatial expression patterns than existing methods. Furthermore, genes detected by

35 SpaGCN are transferrable and can be utilized to study spatial variation of gene expression in other

36 datasets. SpaGCN is computationally fast, making it a desirable tool for spatial transcriptomics studies. 


\section{Introduction}

39 Recent advances in spatial transcriptomics technologies have enabled gene expression profiling with

40 spatial information in tissues ${ }^{1}$. Knowledge of the relative locations of different cells in a tissue is critical

41 for understanding disease pathology because spatial information helps in understanding how the gene

42 expression of a cell is influenced by its surrounding environment and how neighboring regions interact at

43 the gene expression level. Experimental methods to generate spatial transcriptomics data can be broadly

44 classified into two categories: 1) single-molecule fluorescence in situ hybridization (smFISH) based

45 techniques, such as MERFISH${ }^{2}$ and seqFISH ${ }^{3}$, which measure expression level for hundreds of genes with

46 subcellular spatial resolution in a single cell; and 2) spatial barcoding followed by next generation

47 sequencing based techniques, such as SLIDE-seq ${ }^{4}$ and $10 X$ Genomics Visium, which measure the

48 expression level for thousands of genes in captured locations, referred to as spots. These different spatial

49 transcriptomics techniques have made it possible to uncover the complex transcriptional architecture of

50 heterogenous tissues and enhanced our understanding of cellular mechanisms in diseases ${ }^{5,6}$.

52 In spatial transcriptomics studies, an important step is identifying spatial domains defined as regions that

53 are spatially coherent in both gene expression and histology. Identifying spatial domains requires methods

54 that can jointly consider gene expression, spatial location, and histology. Traditional clustering methods

55 such as K-means and Louvain's method ${ }^{7}$ can only take gene expression data as input, and the resulting

56 clusters may not be contiguous due to the lack of consideration of spatial information and histology. To

57 account for spatial dependency of gene expression, new methods have been developed. For example,

58 stLearn ${ }^{8}$ uses features extracted from histology image as well as expression of neighboring spots to

59 spatially smooth gene expression data before clustering; BayesSpace ${ }^{9}$ employs a Bayesian approach for

60 clustering analysis by imposing a prior that gives higher weight to spots that are physically close; Zhu et 
61 al. ${ }^{10}$ uses a Hidden-Markov random field approach to model spatial dependency of gene expression.

62 Although these methods can cluster spots or cells into distinct groups, they do not provide biological 63 interpretations of the identified spatial domains.

65 To link spatial domains with biological functions at the gene expression level, it is crucial to identify genes 66 that show enriched expression in the identified domains. Due to spatial variation of cell types in tissue,

67 the difference of gene expression between different domains is mainly driven by cell type composition 68 variation. On the other hand, information on spatial location and the corresponding histology allows the 69 construction of an anatomy-based taxonomy of the tissue, which provides a useful perspective on cell 70 type composition. Although stLearns integrates gene expression, spatial location, and histology 71 information in clustering, the putative correspondence between cell type difference and organizational 72 structure of the tissue remains unclear. As reported in Saiselet et $a .^{11}$, many spatial regions are highly 73 intermixed in terms of cell types. Without further downstream gene-level analysis, the spatial domains 74 detected by stLearn still suffer from the lack of interpretability. Recently, new methods such as 75 Trendsceek ${ }^{12}$, SpatialDE ${ }^{13}$, and SPARK ${ }^{14}$ have been developed to detect spatially variable genes (SVGs).

76 These methods examine each gene independently and return a $p$-value to represent the spatial variability

77 of a gene. However, due to the lack of consideration of tissue taxonomy, genes detected by these methods 78 do not have a guaranteed spatial expression pattern, making it difficult to utilize these genes for further 79 biological investigations.

81 Rather than considering spatial domain identification and SVG detection as separate problems, we 82 developed SpaGCN, a graph convolutional network-based approach that considers these two problems 83 jointly. Using a graph convolutional network with an added iterative clustering layer, SpaGCN first 84 identifies spatial domains by integrating gene expression, spatial location, and histology together through 
85 the construction of an undirected weighted graph that represents the spatial dependency of the data. For

86 each spatial domain, SpaGCN then detects SVGs that are enriched in the domain against its surrounding

87 regions by differential expression analysis guided by domain information. SpaGCN also has the option to

88 detect meta genes that are uniquely expressed in a given domain. The spatial domains and the

89 corresponding SVGs and meta genes detected for these domains provide a comprehensive picture on the

90 spatial gradients in gene expression in tissue.

92 Results

\section{Overview of SpaGCN and evaluation}

95 SpaGCN is applicable to both sequencing-based and smFISH-based data. As shown in Fig. 1a, SpaGCN first

96 builds a graph to represent the relationship of all samples (spots in sequencing-based or cells in smFISH-

97 based data) considering both spatial location and histology information. Next, SpaGCN utilizes a graph

98 convolutional layer to aggregate gene expression information from neighboring samples. Then, SpaGCN

99 uses the aggregated gene expression matrix to cluster samples using an unsupervised iterative clustering

100 algorithm ${ }^{15}$. Each cluster is considered as a spatial domain from which SpaGCN then detects SVGs that are

101 enriched in a domain by differential expression analysis (Fig. 1b). When a single gene cannot mark

102 expression pattern of a spatial domain, SpaGCN will construct a meta gene, formed by the combination

103 of multiple SVGs, to represent gene expression of the domain. Since the expression profile of a spot/cell

104 is heavily influenced by its local microenvironment, SpaGCN also offers the option of subcluster detection

105 within each spatial domain. SVGs can also be detected to help in understanding the function of each sub-

106 spatial domain.

107

108 To showcase the strength and scalability of SpaGCN, we applied it to five publicly available datasets, 
109 including four datasets generated by sequencing-based techniques and one dataset generated by

110 MERFISH (Supplementary Table 1). The spatial domains identified by SpaGCN agree better with known

111 tissue layer structure than K-means and Louvain's clustering. We also compared SVGs detected by SpaGCN

112 with those detected by SPARK ${ }^{14}$ and SpatialDE ${ }^{13}$, and found that the SVGs detected by SpaGCN have more

113 coherent expression patterns and better biological interpretability than the other two methods. The

114 specificity of spatial expression patterns revealed by SpaGCN detected SVGs were further confirmed by

115 Moran's / statistic ${ }^{16}$, a metric that quantifies the spatial autocorrelation of detected genes.

\section{Application to mouse olfactory bulb data}

118 To evaluate the performance of SpaGCN, we first analyzed a mouse olfactory bulb (MOB) dataset ${ }^{17}$, which

119 consists of 16,218 genes measured in 262 spots. The main olfactory bulb has five layers, ordered from

120 surface to the center as follows: glomerular layer, external plexiform layer, mitral cell layer, internal

121 plexiform layer, and granule cell layer. We compared SpaGCN's clustering results to K-means and Louvain

122 by setting the number of clusters at 5 for all three methods. As shown in Fig. 2a, K-means only identified

1233 main spatial domains, with only few spots assigned to domains 1 and 3 . Louvain's method identified 5

124 main spatial domains. However, since it does not consider spatial and histology information, domains 2 ,

1253 , and 4 have blurred boundaries and more outliers than SpaGCN. By contrast, the domains detected by

126 SpaGCN agree better with the biologically known 5-layer structure of the MOB.

128 To understand the functions of the SpaGCN identified spatial domains, we next detected SVGs for each 129 spatial domain. In total, SpaGCN detected 60 SVGs. Fig. $2 b-f$ shows a randomly selected SVG for each 130 domain, and all genes show strong specificity for the corresponding domain. The In Situ Hybridization 131 labelling of these genes from the Allen Brain Institute further confirmed the correspondence of the spatial 132 domains detected by SpaGCN. Additional SVGs detected by SpaGCN are shown in Supplementary Fig. 1. 
134 As a comparison, we also detected SVGs using SpatialDE and SPARK. SpatialDE identified 67 SVGs, but only

13512 of them overlapped with SpaGCN results (Supplementary Fig. 2). We further looked into the 55 genes

136 detected exclusively by SpatialDE and found many of the genes are expressed in only a few spots or are

137 highly expressed in most of the spots, leading to false detections of significant spatial patterns

138 (Supplementary Fig. 3). By contrast, SpaGCN avoided this issue by filtering out genes using minimum

139 within group expression fraction and maximum between group expression fraction. SPARK detected 772

140 genes, with 49 overlapping with SapGCN (Supplementary Fig. 2). However, we found that the SPARK

141 results indicate that 274 genes have FDR-adjusted p-values less than 0.00001 with 14 of them having the

142 smallest identical FDR-adjusted $p$-value of $4.42 \mathrm{e}-13$. As a result, the SPARK $p$-values are not informative

143 in differentiating the degree of spatial variability between different genes. Of note, none of these 14 genes

144 were detected by SpaGCN. Further examination revealed that some of these genes show spatial variability,

145 but more than half of them are only expressed in a few spots or highly expressed in most of the spots

146 (Supplementary Fig. 4). The FDR-adjusted p-value distribution of SPARK and q-value distribution of

147 SpatialDE are highly skewed toward 0, making it challenging to select informative SVGs based on their p-

148 values or q-values alone (Supplementary Fig. 5).

150 To compare SVGs detected by different methods quantitatively, we calculated the Moran's / statistic, 151 which measures the spatial autocorrelation for each gene. Fig. $2 \mathrm{~g}$ shows the distribution of Moran's $I$.

152 Although all SpaGCN detected SVGs have clear spatial patterns, their Moran's / values are not significantly 153 higher than the SVGs detected by SPARK and SpatialDE (median of 0.20 for SpaGCN against 0.18 for SPARK 154 and 0.25 for SpatialDE). Further examination revealed that many SVGs detected by SPARK and SpatialDE 155 are expressed in multiple adjacent spatial domains. For example, the gene PCP4 uniquely detected by 156 SpatialDE is expressed in two adjacent layers (domains 2 and 4 defined by SpaGCN) (Supplementary Fig. 
157 6). By contrast, all the SVGs detected by SpaGCN are domain specific, offering interpretation in alignment

158 with our knowledge of layer structure. We note that less informative SVGs with clear, but non-domain

159 specific, spatial patterns, such as PCP4, can also be detected by SpaGCN if the user combines domains 2

160 and 4 as the target domain in SVG detection.

161

162 Application to mouse posterior brain data

163 Next, we analyzed a dataset generated from mouse posterior cerebrum, cerebellum and brainstem by 164 10X Genomics that includes 3,353 spots and 31,053 genes $^{18}$. We compared the clustering results of

165 SpaGCN with K-means and Louvain's clustering. The number of clusters in K-means and resolution in 166 Louvain were set to generate the same number of clusters as SpaGCN (10 clusters). Fig. 3a shows that 167 Louvain's clustering is similar to SpaGCN, but the spatial domains detected by SpaGCN are more spatially 168 contiguous than Louvain's results. The integrity of SpaGCN's spatial domains stems from the aggregation 169 of gene expression based on spatial information and histology, which ensures that the genes detected by 170 differential expression analysis have clear spatial expression patterns.

172 SpaGCN detected 523 SVGs for the 10 spatial domains while SPARK and SpatialDE detected 9,678 and

17312,676 SVGs, respectively (Supplementary Fig. 7). We hypothesized that the substantially larger number

174 of SVGs detected by SPARK and SpatialDE are due to the lack of spatial expression patterns that exist in 175 the data. To confirm this hypothesis, we calculated the Moran's / statistic for all detected SVGs (Fig. 3b). 176 The Moran's I values of SpaGCN detected SVGs are much higher than those detected by SPARK and 177 SpatialDE (median of 0.50 for SpaGCN against 0.21 for SPARK and 0.16 for SpatialDE). Closer examination 178 of the SVGs detected by SPARK and SpatialDE revealed that most of the SVGs suffer from one of the two 179 problems observed previously in the MOB dataset: they are (1) only expressed in a few spots or highly 180 expressed in most of the spots, suggesting high false positive rates for SPARK and SpatialDE or (2) spatially 
181 variable, but expressed in multiple adjacent spatial domains, making it difficult to interpret. Another

182 limitation of these two methods is that the FDR-adjusted $p$-value from SPARK and q-value from SpatialDE

183 are not informative. Genes with similar $p$-values/q-values do not necessarily show similar spatial pattern

184 and a smaller p-value/q-value does not guarantee a better spatial pattern (Supplementary Fig. 8 and

185 Supplementary Fig. 9). The p-value and q-value distributions of SPARK and SpatialDE are highly skewed

186 toward 0 (Supplementary Fig. 10). By contrast, the SVGs detected by SpaGCN were enriched in specific

187 spatial domains (Supplementary Fig. 11) and their expression patterns are transferable to an adjacent

188 tissue slice in the mouse posterior brain (Supplementary Fig. 12). Further, multiple domain adaptive

189 filtering criteria implemented in SpaGCN allow it to eliminate false positive SVGs and ensure all detected

190 SVGs have clear spatial expression patterns.

192 To illustrate why appropriate filtering is important, we use domains 1, 5, and 8 as an example. For each of 193 these domains, SpaGCN detected a single SVG enriched in that region. As shown in Fig. 3c, PVALB is 194 enriched in domain 1, and TRM62 is enriched in domain 8. Although domains 1 and 8 are adjacent to each 195 other, these two SVGs can still well mark these domains. NRGN is a SVG that SpaGCN detected for domains 1965 and 7. The high expression of $N R G N$ in domains 5 and 7 also indicate that these two domains are 197 neuroanatomically similar - both consisting of cortex and the pyramidal layer of the hippocampus. Both 198 the cortex and hippocampus are regions that are on the curved surface of the brain. This posterior brain 199 tissue section has the top part of the curved surface in domain 5 and the bottom part of the curved surface 200 in domain 7. Domains 5 and 7, which would be contiguous in a complete 3D reconstruction, are 201 artifactually separated due to the way the section was cut. Therefore, it is not surprising that in addition 202 to NRGN, SpaGCN also detected many other SVGs, such as APP, ATP6V1G2, CALM2, CHN1, CLSTN1, 203 ARPP21, CYP46A1, DCLK1, LINGO1, and MARCKS, that are highly expressed in both domains 5 and 7 
204 (Supplementary Fig. 11). The unique and powerful SVG detection procedure in SpaGCN ensures that genes

205 like these are not missed.

207 SpaGCN did not identify any SVGs for domain 0. However, we reason that a meta gene, formed by the 208 combination of multiple genes, may better reveal spatial patterns than any single genes. We used domain 2090 as an example to show how SpaGCN can create informative meta genes to mark a spatial domain (Fig.

2103 d). First, by lowering the filtering thresholds, SpaGCN identified $K L K 6$ which is highly expressed in the 211 lower part of domain 0 . Using KLK6 as a starting gene, SpaGCN used a novel approach to find a log-linear

212 combination of gene expression of $K L K 6, M B P$ and $A T P 1 B 1$, which accurately marked the spatial domain

2130. In this meta gene, $K L K 6$ and $M B P$ are considered as positive markers because they are highly expressed

214 in some spots in domain 0 , whereas ATP1B1 is considered a negative marker as it is mainly expressed in

215 regions other than domain 0. Previous studies have shown that $K L K 6$ and MBP expression is restricted to 216 oligodendrocytes, while ATP1B1 is mainly expressed in neurons and astrocytes ${ }^{19}$. This resonates the fact

217 that domain 0 represents white matter which is dominated by oligodendrocytes and has few neuronal cell

218 bodies. Therefore, the genes that make up this meta gene have meaningful biological interpretation.

219 Using this meta gene detection procedure, we also detected meta genes for domains 2, 7, 8 and 9, and

220 found that these meta genes are transferrable to an adjacent tissue slice (Supplementary Fig. 13).

222 The expression profile and biological function of a spot is heavily influenced by its neighboring spots. The

223 surrounding spots can trigger a response pathway or signal the spot to perform certain tasks. Although

224 the spots in one spatial domain detected by SpaGCN are spatially coherent and have similar gene 225 expression patterns, they may still have different functions since their surrounding spots are different. For 226 instance, spots located near the boundary of a spatial domain may have different functions compared to 227 spots located in the inner part of the domain. To learn more about the effect of different neighborhoods 
228 on the spots, we performed sub-domain detection. For example, domain 2 is located in the center of the

229 tissue slice and surrounded by multiple other spatial domains. As a result, the neighboring environment

230 for spots in domain 2 varies. As shown in Fig. 3e, domain 2 was separated into 5 sub-domains which are

231 located either in the center or different boundary regions of domain 2, suggesting that differences in the

232 neighborhoods of spots contribute to within-domain heterogeneity. SVGs detected for each sub-domain

233 can help us understand the gene expression variability of spots within each sub-domain.

235 Application to LIBD human dorsolateral prefrontal cortex data

236 In addition to the datasets described previously, SpaGCN also showed advantage over competing methods

237 when evaluated on the LIBD human dorsolateral prefrontal cortex (DLPFC) data ${ }^{20}$. The LIBD study 238 sequenced 12 slices from DLPFC that spans six neuronal layers plus white matter. We started from 239 analyzing slice 151673 , which includes 3,639 spots and 33,538 genes. As the original publication manually 240 annotated the tissue into 7 layers, for fair comparison, the number of clusters was also set at 7 for SpaGCN, 241 K-means, and Louvain. As shown in Fig. 4a, K-means and Louvain failed to separate the tissue into layers 242 with clear boundary. By contrast, SpaGCN successfully identified layer structures with clear boundaries. 243 The Adjusted Rand Indexes (ARIs) for the SpaGCN, K-means, and Louvain identified domains are 0.42, 0.24, 244 and 0.33 , respectively, suggesting that the SpaGCN results better agree with the manually curated layer 245 structure reported in the original study.

247 To further validate the identified spatial domains, we then detected SVGs. In total, SpaGCN detected 61 248 SVGs, with 53 of them specific to domain 4, which corresponds to the white matter region (Supplementary 249 Fig. 14). Patterns of SVGs for other domains are not very clear. These results indicate that gene expression 250 profiles of spots from white matter are distinct from spots in the neuronal layers, while gene expression 251 differences among the six neuronal layers are much smaller and more difficult to distinguish using 
252 individual marker genes. SVGs detected by SPARK and SpatialDE also suffered from the same problem.

253 SPARK detected 3,187 SVGs with 1,131 of them having FDR-adjusted p-values equal to 0, most of which

254 only marked the white matter region. We also found that the SVGs detected by SPARK lack domain

255 specificity (Supplementary Fig. 15). SpatialDE detected 3,654 SVGs with 806 of them having q-values equal

256 to 0 , but these genes do not necessarily show better spatial pattern than genes with larger q-values

257 (Supplementary Fig. 16). Although SPARK and SpatialDE detected much larger numbers of SVGs than

258 SpaGCN (Supplementary Fig. 17), the genes detected by these two methods lack ability to distinguish

259 different degrees of spatial variability in expression as their $p$-value and q-value distributions are highly

260 skewed toward 0 (Supplementary Fig. 18). Fig. 4b shows that the Moran's I values for SpaGCN detected

261 SVGs are significantly higher than those detected by SpatialDE and SPARK (median of 0.39 for SpaGCN

262 against 0.09 for SPARK and 0.08 for SpatialDE). For 3 out of the 6 neuronal layers, SpaGCN detected a

263 single SVG to mark that region (Fig. 4c). For example, NEFM is enriched in domain 0 (layer 3 ) and PCP4 is

264 enriched in domain 1 (layer 4). Although it is difficult to identify single genes to mark the other neuronal

265 layers, SpaGCN was able to find layer-specific meta genes. As shown in Fig. 4c, the meta gene formed by

$266 K R T 19, M Y L 9, M B P, G F A P$, and SNAP25 for domain 5 is specific to layer 1 . Since layer 1 only has few spots,

267 it is difficult to find a highly enriched gene. However, by adding depleted genes like MBP and SNAP25, the

268 expression pattern in this region is strengthened. Furthermore, the SVGs and meta genes detected by

269 SpaGCN are transferrable to slice 151676 obtained from the same study (Supplementary Fig. 19 and

270 Supplementary Fig. 20).

271

272 To show the SVGs and meta genes detected by SpaGCN are useful for downstream analysis, we performed

273 K-means clustering on slice 151676 using SVGs and meta genes detected from slice 151673 by SpaGCN.

274 Specifically, we selected 2 SVGs or meta genes detected by SpaGCN for each spatial domain, resulting in

27514 features (18 unique genes involved in total) used in K-means clustering. Comparing with manually 
curated layer assignment reported in the original study, this clustering analysis had an ARI of 0.25 (Fig.

277 4d). We performed similar clustering analysis using SVGs detected by SpatialDE and SPARK. When only 278 using their top 18 SVGs, the ARI is only 0.07 for SpatialDE and 0.05 for SPARK. Even when using the 806

279 most significant SpatialDE detected SVGs, the ARI is only 0.14 . When using the 1,114 most significant 280 SPARK detected SVGs, the ARI is 0.15 (Fig. 4e). The ARIs of both SpatialDE and SPARK are much lower than 281 SpaGCN, even though both used many more SVGs than SpaGCN, which further confirmed the lack of 282 spatial expression specificity for genes detected by these methods.

\section{Application to human primary pancreatic cancer tissue}

285 We also analyzed a human primary pancreatic cancer tissue dataset ${ }^{5}$, which includes 224 spots and 16,448 286 genes across 3 manually annotated sections, to show SpaGCN's ability in detecting tumorous regions. The 287 original study identified and annotated the cancer region on the histology image. However, the cancer 288 region detected by their clustering method based on gene expression information alone did not closely 289 match the pathologist annotated cancer region (Fig. 5a). Since the cancer region in the histology image is 290 darker in color than non-cancer regions, it is informative for clustering. To give histology information 291 higher weight, we increased the scaling parameter $s$ in SpaGCN from 1 to 2 when calculating distance 292 between each spot pair. This step ensured that spots in the same dark region in the histology are more 293 likely to be clustered together. Fig. 5a shows that domain 2 detected by SpaGCN has a better 294 correspondence to the cancer region than clusters reported in the original study. In total, SpaGCN 295 detected 12 SVGs, with 3, 8, and 1 SVGs for domains 0, 1, and 2, respectively (Fig. 5b; Supplementary Fig. 296 21). Furthermore, a meta gene using KRT17, MMP11, and SERPINA1 marked the cancer region better than 297 the originally identified SVG KRT17 (Fig. 5c). KRT17 functions as a tumor promoter and regulates 298 proliferation in pancreatic cancer ${ }^{21}$, and $M M P 11$ has been found to be a prognostic biomarker for 299 pancreatic cancer ${ }^{22}$. Our identification of $K R T 17$ and MMP11 as the two positive genes for the cancer 
region agree well with pancreatic cancer biology. SPARK and SpatialDE detected 203 and 163 SVGs,

301 respectively (Supplementary Fig. 22). However, the Moran's / values for their SVGs are much lower than

302 those detected by SpaGCN, suggesting their lack of spatial expression patterns (Fig. 5d).

\section{Application to MERFISH mouse hypothalamus data}

305 Next, we show that SpaGCN can also be applied to smFISH-based data. To this end, we analyzed a MERFISH

306 dataset generated from the preoptic region of hypothalamus in mouse brain ${ }^{2}$, which includes 5,665 cells

307 and 161 genes. One important difference between MERFISH and sequencing-based spatial

308 transcriptomics data is that the captured tissue area is much smaller and less genes are measured, making

309 it difficult to detect spatial domains since the cells within such a small area are more similar to each other.

310 Thus, when utilizing these types of data, we suggest increasing the contribution of neighboring cells when

311 calculating the weighted gene expression of each cell. Using this approach, SpaGCN detected spatial

312 domains that agreed well with the annotated hypothalamic nuclei (Fig. 6a), with domain 2 corresponding

313 to ACA, domain 3 corresponding to PS, and domain 7 corresponding to MnPo. By contrast, the domains

314 identified from the Hidden Markov Random Field (HMRF) approach showed little overlap with the

315 hypothalamic region annotation. Using SpaGCN, we further detected 19 SVGs including DGKK, ERMN, and

$316 S L N$ that showed enriched expression patterns for domains 2, 3, and 7 (Fig. 6b; Supplementary Fig. 23).

\section{Discussion}

319 Identification of spatial domains and detection of SVGs are important steps in spatial transcriptomics data

320 analysis. In this paper, we presented SpaGCN, a graph convolutional network-based approach that

321 integrates gene expression, spatial location, and histology to model spatial dependency of gene

322 expression for clustering analysis of spatial domains and identification of domain enriched SVGs or meta

323 genes. Through the use of a convolutional layer in an undirected weighted graph, SpaGCN aggregates 
324 gene expression of each spot from its neighboring spots, which enables the identification of spatial

325 domains with coherent gene expression and histology. The subsequent domain guided differential

326 expression analysis also enables the detection of SVGs or meta genes with enriched expression patterns

327 in the identified domains. SpaGCN has been extensively tested on datasets from different species, regions,

328 and tissues generated using both sequencing- and smFISH-based techniques. The results consistently

329 showed that SpaGCN can identify spatial domains with coherent gene expression and histology and detect

330 SVGs and meta genes that have much clearer spatial expression patterns and biological interpretations

331 than genes detected by SPARK and SpatialDE. Additionally, the SpaGCN detected SVGs and meta genes

332 are transferrable and can be utilized for downstream analyses in independent tissue sections.

334 The spatial domain detection step in SpaGCN is flexible. For datasets with clear layer structure in histology

335 image, such as the mouse posterior brain data and human primary pancreatic cancer data, higher weight

336 can be given to histology by increasing the scaling parameter $s$ in SpaGCN when calculating distance

337 between each spot pair, which results in spatial domains that are more similar to the anatomy-based

338 taxonomy in the histology image. Another important scaling parameter in SpaGCN is the characteristic

339 length scale $l$, which controls the relative contribution from other spots when aggregating gene

340 expression. By varying $l$, users can get spatial domain separations with different patterns in which a higher

$341 \quad l$ will result in spatial domains with higher contiguity.

343 The SVG detection procedure in SpaGCN is also flexible. While we mainly demonstrated SVG detection for 344 a single domain, SpaGCN also allows users to combine multiple domains as one target domain or specify 345 which neighboring domains to be included in DE analysis. Additionally, SpaGCN allows the users to 346 customize SVG filtering criteria based on $p$-value and three additional metrics, i.e., in-fraction, in/out 
347 fraction ratio, and fold change, to select SVGs. The resulting genes can be ranked by any of these metrics

348 to select SVGs with desired spatial expression patterns.

350 SpaGCN is computationally fast and memory efficient. To showcase the computational advantage of

351 SpaGCN, we recorded its run time and memory usage for the mouse posterior brain data and compared

352 with SPARK and SpatialDE. All analyses were conducted on Mac OS 10.13 .6 with single Intel ${ }^{\circledR}$ Core(TM) i5-

353 8259U CPU @2.30GHz and 16GB memory. As shown in Supplementary Fig. 24, SpaGCN completed spatial

354 domain and SVG detection in less than one minute, whereas the computing time is 13 minutes for

355 SpatialDE and more than 18 hours for SPARK. Furthermore, SpaGCN only required 1.3 GB of memory,

356 whereas SpatialDE and SPARK required more than $3.1 \mathrm{~GB}$ and 7.2 GB of memory, respectively. With the

357 increasing popularity of spatial transcriptomics in biomedical research, we expect SpaGCN will be an

358 attractive tool for large-scale spatial transcriptomics data analysis. Results from SpaGCN will enable 359 researchers to accurately identify spatial domains and SVGs in their studies.

\section{Acknowledgements}

362 This work was supported by the following grants: R01GM125301, R01EY030192, R01EY031209 363 R01HL113147, and R01HL150359 (to M.L.), and P01AG066597 (to D.J.I. and E.B.L.). We thank Reuben 364 Moncada and Itai Yanai for sharing the human pancreatic cancer histology image data.

\section{Author contributions}

367 This study was conceived of and led by M.L.. J.H. designed the model and algorithm. J.H. implemented the 368 SpaGCN software and led the data analysis with input from M.L., X.L., K.C., A.S., D.I., E.L., and R.T.S.. J.H. 369 and M.L. wrote the paper with feedback from all other coauthors. 


\section{Competing financial interests}

372 The authors declare no competing interests. 


\section{Figure legends}

375 Figure 1. Workflow of SpaGCN. a, SpaGCN starts from integrating gene expression, spatial location and 376 histology information using a graph convolutional network (GCN), then separates spots into different 377 spatial domains using unsupervised iterative clustering. The GCN is based on an undirected weighted 378 graph in which the edge weight between every two spots is determined by Euclidean distance between 379 the two spots, defined by the spatial coordinates $(x, y)$ and the 3-rd dimensional coordinate $z$, obtained 380 from the RGB values in the histology image. $\mathbf{b}$, For each detected spatial domain, SpaGCN identifies SVGs 381 or meta genes by domain guided differential expression analysis.

383 Figure 2. Spatial domains and SVGs detected in the mouse olfactory bulb dataset. a, Histology image of 384 the tissue section and spatial domains detected by SpaGCN, Louvain's method, and K-means clustering. 385 b-f, Spatial expression patterns of SVGs detected by SpaGCN for domains 0 (LCAT), 1 (NR2F2), 2 (CACNB3), 3863 (SLC17A7), and 4 (NECAB2), and the corresponding in situ hybridization of these SVGs obtained from the 387 Allen Brain Atlas. g, Boxplot of Moran's / values for SVGs detected by SpaGCN, SPARK, and SpatialDE.

Figure 3. Spatial domains and SVGs detected in the mouse brain posterior brain dataset. a, Histology

390 image of the tissue section and spatial domains detected by SpaGCN, Louvain's method, and K-means 391 clustering. b, Boxplot of Moran's I values for SVGs detected by SpaGCN, SPARK, and SpatialDE. c, Spatial 392 expression patterns of SVGs detected by SpaGCN for domain 1 (PVALB), 8 (TRIM62), and 5 (NRGN). d, 393 Spatial expression patterns of genes $K L K 6, M B P, A T P 1 B 1$, which form the specific marker meta gene for 394 domain $0(K L K 6+M B P-A T P 1 B 1)$. e, Clustering results for 5 sub-domains detected by SpaGCN for domain 3952 , and the spatial expression patterns of SVGs for sub-domains 0 (KCNC3), 1 (CAMK2A), and 2 (NRSN2). 
398 a, Manually annotated layer structure for slice 151673 from the original study ${ }^{20}$, and spatial domains 399 detected by SpaGCN, Louvain's method, and K-means clustering. b, Boxplot of Moran's / values for SVGs

400 detected by SpaGCN, SPARK, and SpatialDE for slice 151673. c, Spatial expression patterns of SVGs for 401 domain 0 (NEFM) and domains 1 (PCP4), and a meta gene formed by KRT19, MYL9, MBP, GFAP, and 402 SNAP25 for domain $5(K R T 19+$ MYL9 - MBP + GFAP - SNAP25). d, Manually annotated layer structure for 403 slice 151676 from the original study ${ }^{20}$, and K-means clustering results for slice 151676 using 18 genes 404 selected by SpaGCN, SPARK, and SpatialDE. For SpaGCN, we selected the following genes, domain 0 (NEFL, 405 NEFM), domain 1 (PCP4, TMSB10 + PCP4 - KRT19), domain $2(C C K+K R T 17-M T-N D 1, C P L X 2+K R T 17-$ 406 MT-ND2), domain $3(C A M K 2 N 1, E N C 1)$, domain $4(M B P, F T L)$, domain $5(K R T 19+M Y L 9-M B P+G F A P-$ $407 P L P 1, K R T 8+M Y L 9-M B P+G F A P-P L P 1)$, and domain $6(G F A P, M B P)$, resulting in 18 unique genes in 408 total. For SPARK and SpatialDE, the 18 SVGs with the smallest FDR-adjusted p-value or q-value were 409 randomly selected. e, ARIs between manually annotated layers and K-means' clustering using SVGs 410 selected by different methods. For SpaGCN, we only used the selected SVGs and meta genes, with 18 411 genes involved in total while for SPARK and SpatialDE, we used top 18, 100, 200, 500 and all SVGs with 412 the identical smallest FDR-adjusted $p$-value or q-value.

414 Figure 5. Spatial domains and SVGs detected in the human primary pancreatic cancer tissue dataset. a, 415 Histology image of the tissue section with manually annotated regions from the original study ${ }^{5}$, spatial 416 domains detected by SpaGCN, and clustering results from the original study. $\mathbf{b}$, Spatial expression pattern 417 of SVGs detected by SpaGCN for domain 0 (AEBP1) and domain 1 (SERPINA1). c, Spatial expression 418 patterns of genes KRT17, MMP11, SERPINA1, which form the specific marker meta gene for domain 2 419 (KRT17 + MMP11 - SERPINA1). d, Boxplot of Moran's / values for SVGs detected by SpaGCN, SPARK, and 420 SpatialDE. 
422 Figure 6. Spatial domains and SVGs detected in the MERFISH mouse brain hypothalamus dataset. a,

423 Spatial domains detected by SpaGCN and the HMRF method overlayed with annotated hypothalamic

424 nuclei from the original study ${ }^{2}$, and cell type distribution from the original study. d, Spatial expression

425 patterns of SVGs detected by SpaGCN for domain 2 (ERMN), domain $3(D G K K)$, and domain 7 (SLN). 


\section{Data preprocessing}

429 SpaGCN takes spatial gene expression and histology image data (when available) as input. The spatial gene 430 expression data are stored in an $N \times D$ matrix of unique molecular identifier (UMI) counts with $N$ samples

431 and $D$ genes, along with the $(x, y)$ 2-dimensional spatial coordinates of each sample. In sequencing-based 432 data, each sample represents a spot containing multiple cells, whereas in single-molecule fluorescence in 433 situ hybridization (smFISH)-based data, each sample represents a single cell. For simplicity, we will use 434 'spot' to refer to a sample, as most of the data analyzed in this paper are sequencing based. Genes 435 expressed in less than three spots are eliminated. The gene expression values in each spot are normalized 436 such that the unique molecular identifier (UMI) count for each gene is divided by the total UMI count 437 across all genes in a given spot, multiplied by 10,000 , and then transformed to a natural log scale.

\section{Conversion of spatial transcriptomics data into graph-structured data}

440 After preprocessing, SpaGCN converts the gene expression and histology image data into a weighted 441 undirected graph, $G(V, E)$. In this graph, each vertex $v \in V$ represents a spot and every two vertices in $V$ 442 are connected via an edge with a specified weight. We focus our analysis on spatial transcriptomics data 443 with histology information, but the method can be easily adapted to analyze smFISH-based data, for which 444 histology information is not available.

\section{Calculation of distance between two vertices}

447 The distance between any two vertices $u$ and $v$ in the graph reflects the relative similarity of the two 448 corresponding spots. This distance is determined by two factors: 1 ) the physical locations of spots $u$ and $449 v$ in the tissue slice, and 2) the corresponding histology information of these two spots. Although some 
450 spots are physically close to each other in the tissue, the histology image may reveal that they belong to

451 different tissue layers. Therefore, SpaGCN considers two spots to be close if and only if 1) the two spots

452 are physically close, and 2) they have similar pixel features as shown in the histology image. To define a

453 distance metric considering both aspects, SpaGCN extends the 2-dimensional space in the tissue slice into

454 a 3-dimensional space that incorporates histology information. For spot $v$, its physical location in the

455 tissue slice is represented by 2-dimensional coordinates $\left(x_{v}, y_{v}\right)$. To determine the corresponding pixel in

456 the histology image for spot $v, \operatorname{SpaGCN}$ maps spot $v$ to the histology image according to its pixel

457 coordinates $\left(x_{p v}, y_{p v}\right)$. Instead of using the color of the pixel at $\left(x_{p v}, y_{p v}\right)$, SpaGCN draws a square

458 centered on $\left(x_{p v}, y_{p v}\right)$ containing $50 \times 50$ pixels and calculates the mean color value for the RGB

459 channels, $\left(r_{v}, g_{v}, b_{v}\right)$, of all pixels that fall in the square. This step smooths the color value and ensures

460 that the color is not dominated by a single pixel. To derive a single value to represent the histology image

461 features, SpaGCN uses a weighted sum of the RGB values as follows,

462

$$
z_{v}=\frac{r_{v} \times V_{r}+g_{v} \times V_{g}+b_{v} \times V_{b}}{V_{r}+V_{g}+V_{b}}
$$

465 where $V_{r}=\operatorname{Variance}\left(r_{v}\right), V_{g}=\operatorname{Variance}\left(g_{v}\right)$, and $V_{b}=\operatorname{Variance}\left(b_{v}\right)$ for all $v \in V$. In this transformation, higher weight is given to the channel with larger variance so that this combined value $z_{v}$

467 captures an accurate representation of the patterns in the histology image.

468

$$
z_{v}^{*}=\frac{z_{v}-\mu_{z}}{\sigma_{z}} \times \max \left(\sigma_{x}, \sigma_{y}\right) \times s
$$


473 where $\mu_{z}$ is the mean of $z_{v}, \sigma_{x}, \sigma_{y}, \sigma_{z}$ are the standard deviations of $x_{v}, y_{v}$ and $z_{v}$, respectively, for $v \in$

$474 V$, and $s$ is a scaling factor. In our analysis, $s$ is usually set at 1 to make sure that $z_{v}^{*}$ has the same scale 475 variance as $x_{v}$ and $y_{v}$, and we set $s$ to a value larger than 1 when the goal is to increase the weight of 476 histology. The coordinates of spot $v$ are set to be $\left(x_{v}, y_{v}, z_{v}^{*}\right)$ in the extended 3-dimensional space. Finally,

477 the Euclidean distance between every two spots $u$ and $v$ is calculated as

$$
d(u, v)=\sqrt{\left(x_{u}-x_{v}\right)^{2}+\left(y_{u}-y_{v}\right)^{2}+\left(z_{u}^{*}-z_{v}^{*}\right)^{2}} .
$$

\section{Calculation of weight for each edge and construction of graph}

482 The weight of each edge $(u, v)$ measures the degree of relatedness between spots $u$ and $v$ and is 483 negatively associated with their distance. The graph structure $G$ is stored in an $N \times N$ adjacency matrix $484 \boldsymbol{A}=[w(u, v)]$, where the edge weight between spot $u$ and spot $v$ and is defined as

$$
w(u, v)=\exp \left(-\frac{d(u, v)^{2}}{2 l^{2}}\right)
$$

The hyperparameter $l$, also known as the characteristic length scale, determines how rapidly the weight decays as a function of distance. A similar function has been employed in SpatialDE ${ }^{13}$. Let $\boldsymbol{I}$ denote the

490 identity matrix. For spot $\boldsymbol{v}$, the corresponding row sum of $\boldsymbol{A}-\boldsymbol{I}$, denoted by $a_{v}$, can be interpreted as the 491 relative contribution of other spots to its gene expression. We choose the value of $l$ such that the average 492 of $a_{v}$ across all spots is equal to a pre-specified value, e.g. 0.5 . 
495 SpaGCN reduces the dimension of the preprocessed gene expression matrix using principal component

496 analysis (PCA). The top 50 principal components are used as input, which work well for all datasets

497 analyzed in this paper. Next, utilizing the power of a graph convolutional network, SpaGCN concatenates

498 the gene expression information and edge weights in $G$ to cluster the nodes. Following Kipf and Welling ${ }^{23}$,

499 the graph convolutional layer can be written as

$$
f(\boldsymbol{X}, \boldsymbol{A})=\delta(\boldsymbol{A} \boldsymbol{X} \boldsymbol{B})
$$

503 where $\boldsymbol{X}$ is the $N \times 50$ embedding matrix obtained from PCA, $\boldsymbol{B}$ is a $50 \times 50$ matrix representing filter 504 parameters of the convolutional layer, and $\delta(\cdot)$ is a non-linear activation function such as ReLU. The graph 505 convolutional layer ensures that a corresponding row of parameters in $\boldsymbol{B}$ will control the aggregation of 506 neighborhood information for each feature in $\boldsymbol{X}$, thus offering the flexibility of feature specific aggregation

507 of information provided by neighboring spots. The filter parameters in $\boldsymbol{B}$ are shared across all vertices in 508 the graph and are automatically updated during an iterative training progress. Through graph convolution, 509 SpaGCN has aggregated the gene expression information according to the edge weights specified in $G$.

510 The output of this layer is an aggregated matrix that includes information on gene expression, spatial 511 location, and histology. The graph convolutional layer was implemented based on Kipf and Welling ${ }^{23}$, 512 where the backpropagation is operated via a localized first-order approximation of spectral graph 513 convolution.

\section{Spatial domain identification by clustering}

516 Next, based on the output from the above graph convolutional layer, SpaGCN employs an unsupervised 517 clustering algorithm to iteratively cluster the spots into different spatial domains ${ }^{15}$. Each cluster identified 518 from this analysis is considered to be a spatial domain, which contains spots that are coherent in gene 
519 expression and histology. To initialize cluster centroids, we use Louvain's method ${ }^{7}$ on the aggregated 520 output matrix from the graph convolutional layer. If the number of domains in the tissue is known, the 521 resolution parameter in Louvain will be set to generate the same number of spatial domains. Otherwise, 522 we vary the resolution parameter from 0.2 to 1.0 and select the resolution that gives the highest 523 Silhouette score ${ }^{24}$.

525 To update the cluster assignments iteratively, we define a metric to measure the distance from a spot to 526 a cluster centroid using the Student's $t$-distribution as a kernel. The distance between the embedded 527 point $h_{i}$ for spot $i$ and centroid $\mu_{j}$ for cluster $j$

$$
q_{i j}=\frac{\left(1+\left\|h_{i}-\mu_{j}\right\|^{2}\right)^{-1}}{\sum_{j^{\prime}=1}^{K}\left(1+\left\|h_{i}-\mu_{j^{\prime}}\right\|^{2}\right)^{-1}},
$$

can be interpreted as the probability of assigning cell $i$ to cluster $j$.

$$
p_{i j}=\frac{q_{i j}^{2} / \sum_{i=1}^{N} q_{i j}}{\sum_{j^{\prime}=1}^{K}\left(q_{i j^{\prime}}^{2} / \sum_{i=1}^{N} q_{i j^{\prime}}\right)}
$$

537 which upweights spots assigned with high confidence, and normalizes the contribution of each centroid

538 to the overall loss function to prevent large clusters from distorting the hidden feature space. Now that 539 we have the soft assignment $q_{i j}$ and the auxiliary distribution $p_{i j}$, we can define the objective function as 540 a Kullback-Leibler (KL) divergence loss, 


$$
L=K L(P \| Q)=\sum_{i=1}^{N} \sum_{j=1}^{K} p_{i j} \log \frac{p_{i j}}{q_{i j}} .
$$

544 The network parameters and cluster centroids are simultaneously optimized by minimizing $L$ using 545 stochastic gradient descent with momentum. This unsupervised iterative clustering algorithm has been 546 previously utilized for scRNA-seq analysis and showed superior performance over Louvain's method ${ }^{25,26}$.

\section{Detection of spatially variable genes}

549 We are interested in detecting spatially variable genes (SVGs) that are enriched in each spatial domain.

550 We note that some genes may be expressed in multiple but disconnected domains. Although they are not

551 uniquely expressed in a particular domain, these genes are still useful for understanding spatial variation

552 of gene expression and can be used to form meta genes that are uniquely expressed in a specific domain.

553 Therefore, rather than doing differential expression (DE) analysis using spots from a target domain versus

554 all other spots, we first select spots to form a neighboring set of the target domain. The goal is to detect

555 genes that are highly expressed in the target domain but are not expressed or are expressed at low levels

556 in the neighboring spots. To determine which spots should be considered as neighbors, we draw a circle

557 with a prespecified radius around each spot in the target domain. All spots from non-target domains that

558 reside in the circle are considered its neighbors. The radius is set such that all spots in the target domain

559 have approximately 8 neighbors on average. Next, neighbors of all spots in the target domain are collected

560 and form a neighboring set. For each non-target domain, if more than $50 \%$ (default) of its spots are in the

561 neighboring set, this domain is then selected as a neighboring domain. This criterion is set to avoid the

562 situation when a domain is selected as a neighboring domain, but only a small proportion of its spots are

563 adjacent to the target domain. 
565 After neighboring domains are determined, SpaGCN then performs DE analysis between spots in the

566 target domain and the neighboring domain(s) using Wilcoxon rank-sum test. Genes with a false discovery

567 rate (FDR) adjusted $p$-value $<0.05$ are selected as SVGs. To ensure only genes with enriched expression

568 patterns in the target domain are selected, we further require a gene to meet the following three criteria:

569 1) the percentage of spots expressing the gene in the target domain, i.e., in-fraction, is $>80 \%$; 2 ) for each

570 neighboring domain, the ratio of the percentages of spots expressing the gene in the target domain and

571 the neighboring domain(s), i.e., in/out fraction ratio, is >1; and 3) the expression fold change between the

572 target and neighboring domain(s) is $>1.5$. If a user is interested in finding SVGs for a particular combination

573 of spatial domains, SpaGCN offers the option to do so.

575 Detection of spatially variable meta genes

576 The spatial domain-specific DE analysis described above typically detects SVGs with enriched expression

577 for the majority of the domains. For domains in which no such SVGs are detected, we aim to identify a set

578 of genes that, when combined to form a meta gene, shows an enriched expression pattern in the given

579 domain. To identify genes to form a meta gene, we employ a multi-step approach. First, we lower the

580 thresholds for SVG filtering, e.g., change the minimum fold change threshold from 1.5 to 1.2 , to identify

581 genes showing weaker enriched expression pattern in the target domain. In the presence of multiple such

582 weaker SVGs, we randomly select one of them as the base gene and denote it as gene $e_{0}$. Second, we aim

583 to aggregate expression from other genes to the base gene to enhance the spatial pattern for the target

584 domain. To achieve this goal, we first calculate the mean expression level of gene $_{0}$ for spots in the target

585 domain as $e_{0}$. Then, all spots from non-target domains with $g e n e_{0}$ 's expression level higher than $e_{0}$ are

586 extracted to form a control group. Next, we perform DE analysis using spots from the target domain

587 against spots in the control group using Wilcoxon rank-sum test. The gene with the smallest FDR-adjusted

588 p-value and higher expression in the target domain is selected as gene $_{0+}$. Similarly, we perform DE 
analysis using spots from the control group against those from the target domain and select a gene with

590 the smallest FDR-adjusted p-value and higher expression in the control group as gene $0_{--}$. The meta gene's

591 expression is calculated as

$$
\log \left(\text { meta_gene }_{1}\right)=\log \left(\text { gene }_{0}\right)+\log \left(\text { gene }_{0+}\right)-\log \left(\text { gene }_{0-}\right)+C_{0},
$$

where $C_{0}$ is a constant to make $\log \left(\right.$ meta $\left._{-} g e n e_{1}\right)$ non-negative. The log transformation is used to rescale expression and make the expression levels comparable across different genes. We have found that including negative genes can strengthen spatial expression pattern for domains that do not have enriched positive marker genes. This algorithm can be used iteratively to find additional genes to form an updated meta gene with a clearer spatial pattern for the target domain. For the $(t+1)^{t h}$ iteration, the meta gene expression is calculated as

$$
\log \left(\text { meta_gene }_{t+1}\right)=\log \left(\text { meta }_{-} \text {gene }_{t}\right)+\log \left(\text { gene }_{t+}\right)-\log \left(\text { gene }_{t-}\right)+C_{t}
$$

604 In the $(t+1)^{t h}$ iteration, after adding gene $_{t+}$ and subtracting gene $e_{t-}$, SpaGCN will select the $(t+1)^{t h}$ 605 control group based on meta_gene $e_{t+1}$. The size of the new control group, which is the number of spots 606 not in the target domain but have higher expression of meta_gene $e_{t+1}$ than spots in the target domain, 607 should be smaller than the size of the $t^{t h}$ control group, to ensure that meta_gene $_{t+1}$ has a clearer

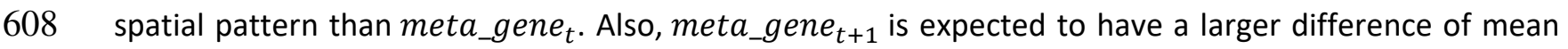
609 expression between the target and control groups than meta_gene ${ }_{t}$. Therefore, at each iteration, 610 SpaGCN checks whether both criteria are met, and the search of additional genes will stop otherwise. An 611 illustration of this iterative meta gene search is shown in Supplementary Fig. 25. 


\section{Evaluation of spatially variable genes using Moran's / statistic}

614 The Moran's / statistic ${ }^{16}$ is a measure of spatial autocorrelation, which can be used to measure the degree

615 of spatial variability in gene expression ${ }^{27}$. The Moran's / value ranges from -1 to 1 , where a value close to

6161 indicates a clear spatial pattern, a value close to 0 indicates random spatial expression, and a value close

617 to -1 indicates a chess board like pattern. To evaluate the spatial variability of a given gene, we calculate

618 the Moran's / using the following formula,

619

$$
I=\frac{N}{W} \frac{\sum_{i} \sum_{j}\left[w_{i j}\left(x_{i}-\bar{x}\right)\left(x_{j}-\bar{x}\right)\right]}{\sum_{i}\left(x_{i}-\bar{x}\right)^{2}},
$$

where $x_{i}$ and $x_{j}$ are gene expression of spots $i$ and $j, \bar{x}$ is the mean expression of the gene, $N$ is the total

623 number of spots, $w_{i j}$ is spatial weight between spots $i$ and $j$ calculated using the 2 -dimensional spatial

624 coordinates of the spots, and $W$ is the sum of $w_{i j}$. For each spot, we select the $k$ nearest neighbors using

625 spatial coordinates. Moran's / statistic is robust to the choice of $k$ and is set at 4 in our analysis. We assign

$626 w_{i j}=1$ if spot $j$ is in the nearest neighbors of spot $i$, and $w_{i j}=0$ otherwise.

\section{Detection of subclusters within a spatial domain}

629 To better characterize heterogeneity within a spatial domain due to the influence of its neighborhood,

630 SpaGCN can further detect sub-domains within each spatial domain by utilizing information from

631 neighboring spots. SpaGCN draws a circle around each spot with a pre-specified radius, and all spots that

632 reside in the circle are considered as neighbors of this spot. The value of the radius is set to ensure that

633 every spot in the target domain have ten neighbors on average. Next, SpaGCN records the number of

634 neighbors from different spatial domains for each spot and stores this information in a $T \times K$ matrix,

635 where $T$ is the number of spots in the target domain and $K$ is the total number of spatial domains 
636 detected. The value for the $i^{\text {th }}$ row and $j^{\text {th }}$ column is the number of neighbors of spot $i$ belonging to

637 domain $j$. Next, this matrix is fed into a $K$-means classifier to detect sub-clusters. Differential expression

638 analysis as described above can be performed to identify subcluster enriched genes.

640 Data availability

641 We analyzed multiple spatial transcriptomics datasets. Publicly available data were acquired from the

642 following websites or accession numbers: (1) mouse olfactory bulb

643 (https://drive.google.com/drive/folders/1C4I3|BaYI7uuV2AA2o0WDzO mkc b0pv?usp=sharing); (2)

644 mouse posterior brain (https://support.10xgenomics.com/spatial-gene-

645 expression/datasets/1.0.0/V1 Mouse Brain Sagittal Posterior); (3) LIBD human dorsolateral prefrontal

646 cortex Dorsolateral pre-frontal cortex (http://research.libd.org/spatialLIBD/); (4) human primary

647 pancreatic cancer data (GSE111672); (5) MERFISH mouse hypothalamus data

648 (https://datadryad.org/stash/dataset/doi:10.5061/dryad.8t8s248). Details of the datasets analyzed in

649 this paper were described in Supplementary Table 1.

650

651 Software availability

652 An open-source implementation of the SpaGCN algorithm can be downloaded from 653 https://github.com/jianhuupenn/SpaGCN

654

\section{Life sciences reporting summary}

656 Further information on experimental design is available in the Life Sciences Reporting Summary.

\section{References}


659 1. Asp, M., Bergenstrahle, J. \& Lundeberg, J. Spatially resolved transcriptomes-next generation 660 tools for tissue exploration. Bioessays 42, e1900221 (2020).

661 2. Moffitt, J.R., et al. Molecular, spatial, and functional single-cell profiling of the hypothalamic 662 preoptic region. Science 362, eaau5324 (2018).

663 3. Eng, C.L., et al. Transcriptome-scale super-resolved imaging in tissues by RNA seqFISH. Nature $664 \quad 568,235-239$ (2019).

665 4. Rodriques, S.G., et al. Slide-seq: A scalable technology for measuring genome-wide expression at 666 high spatial resolution. Science 363, 1463-1467 (2019).

667 5. Moncada, R., et al. Integrating microarray-based spatial transcriptomics and single-cell RNA-seq 668 reveals tissue architecture in pancreatic ductal adenocarcinomas. Nat Biotechnol 38, 333-342 669 (2020).

$670 \quad 6 . \quad$ Chen, W.T., et al. Spatial transcriptomics and in situ sequencing to study Alzheimer's disease. $671 \quad$ Cell 182, 976-991 e919 (2020).

672 7. Blondel, V.D., Guillaume, J.-L., Lambiotte, R. \& Lefebvre, E. Fast unfolding of communities in 673 large networks. Journal of Statistical Mechanics: Theory and Experiment 2008, P10008 (2008).

674 8. Pham, D., et al. stLearn: integrating spatial location, tissue morphology and gene expression to 675 find cell types, cell-cell interactions and spatial trajectories within undissociated tissues. bioRxiv $676 \quad$ (2020).

677 9. Zhao, E., et al. BayesSpace enables the robust characterization of spatial gene expression 678 architecture in tissue sections at increased resolution. bioRxiv (2020).

679 10. Zhu, Q., Shah, S., Dries, R., Cai, L. \& Yuan, G.C. Identification of spatially associated 680 subpopulations by combining scRNAseq and sequential fluorescence in situ hybridization data. $681 \quad$ Nat Biotechnol 36, 1183-1190 (2018). 
682 11. Saiselet, M., et al. Transcriptional output, cell types densities and normalization in spatial

683 transcriptomics. J Mol Cell Biol, mjaa028 (2020).

684 12. Edsgard, D., Johnsson, P. \& Sandberg, R. Identification of spatial expression trends in single-cell gene expression data. Nat Methods 15, 339-342 (2018).

686 13. Svensson, V., Teichmann, S.A. \& Stegle, O. SpatialDE: identification of spatially variable genes. Nat Methods 15, 343-346 (2018).

14. Sun, S., Zhu, J. \& Zhou, X. Statistical analysis of spatial expression patterns for spatially resolved transcriptomic studies. Nat Methods 17, 193-200 (2020).

690 15. Xie, J., Girshick, R. \& Farhadi, A. Unsupervised deep embedding for clustering analysis. Proceedings of the 33rd International Conference on Machine Learning 48(2016).

692 16. Li, H., Calder, C.A. \& Cressie, N. Beyond Moran's I: testing for spatial dependence based on the spatial autoregressive model. Geographical Analysis 39, 357-375 (2007).

694 17. Stahl, P.L., et al. Visualization and analysis of gene expression in tissue sections by spatial transcriptomics. Science 353, 78-82 (2016).

696 18. Dataset. https://support.10xgenomics.com/spatial-geneexpression/datasets/1.0.0/V1 Mouse Brain Sagittal Posterior. (2020).

698 19. Zhang, Y., et al. Purification and Characterization of Progenitor and Mature Human Astrocytes 699 Reveals Transcriptional and Functional Differences with Mouse. Neuron 89, 37-53 (2016).

700 20. Maynard, K.R., et al. Transcriptome-scale spatial gene expression in the human dorsolateral 701 prefrontal cortex. bioRxiv (2020).

702 21. Li, D., et al. KRT17 Functions as a Tumor Promoter and Regulates Proliferation, Migration and 703 Invasion in Pancreatic Cancer via mTOR/S6k1 Pathway. Cancer Manag Res 12, 2087-2095 (2020).

704 22. Lee, J., Lee, J. \& Kim, J.H. Identification of Matrix Metalloproteinase 11 as a Prognostic 705 Biomarker in Pancreatic Cancer. Anticancer Res 39, 5963-5971 (2019). 
706 23. Kipf, T.N. \& Welling, M. Semi-supervised classification with graph convolutional networks.

707 International Conference on Learning Representations arXiv:1609.02907(2017).

708 24. Rousseeuw, P.J. Silhouettes: a graphical aid to the interpretaion and validation of cluster

709 analysis. Computational and Applied Mathematics 20, 53-65 (1987).

$71025 . \quad \mathrm{Li}, \mathrm{X}$., et al. Deep learning enables accurate clustering with batch effect removal in single-cell

$711 \quad$ RNA-seq analysis. Nat Commun 11, 2338 (2020).

712 26. Lakkis, J., et al. A joint deep learning model for simultaneous batch effect correction, denoising 713 and clustering in single-cell transcriptomics. bioRxiv (2020).

714 27. Abdelaal, T., Mourragui, S., Mahfouz, A. \& Reinders, M.J.T. SpaGE: spatial gene enhancement 715 using scRNA-seq. Nucleic Acids Res 48, e107 (2020).

716 

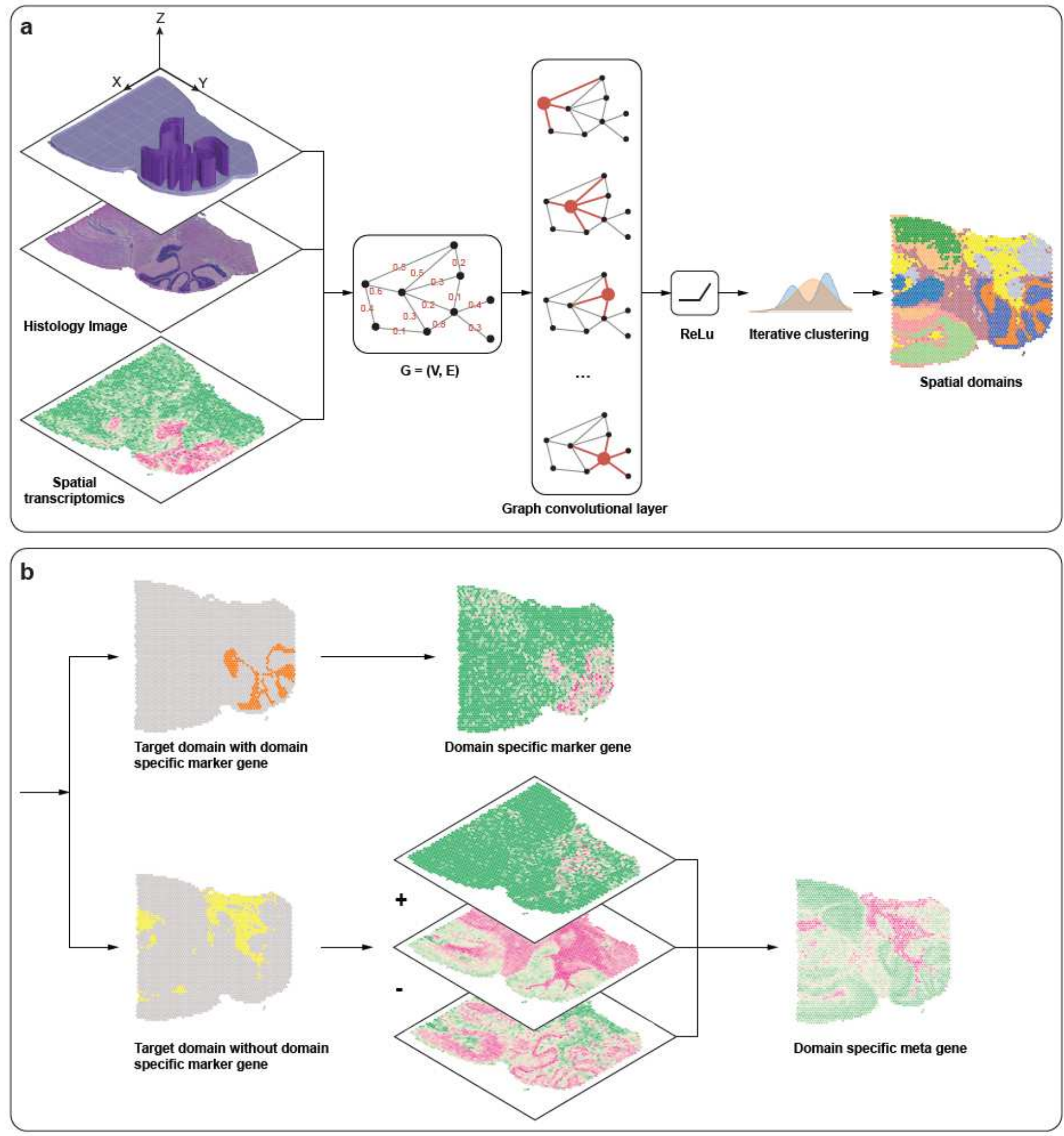

\section{Figure 1}

Workflow of SpaGCN. a, SpaGCN starts from integrating gene expression, spatial location and histology information using a graph convolutional network (GCN), then separates spots into different spatial domains using unsupervised iterative clustering. The GCN is based on an undirected weighted graph in 
which the edge weight between every two spots is determined by Euclidean distance between the two

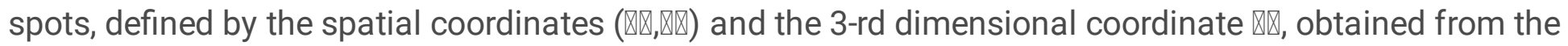
RGB values in the histology image. b, For each detected spatial domain, SpaGCN identifies SVGs or meta genes by domain guided differential expression analysis.

a
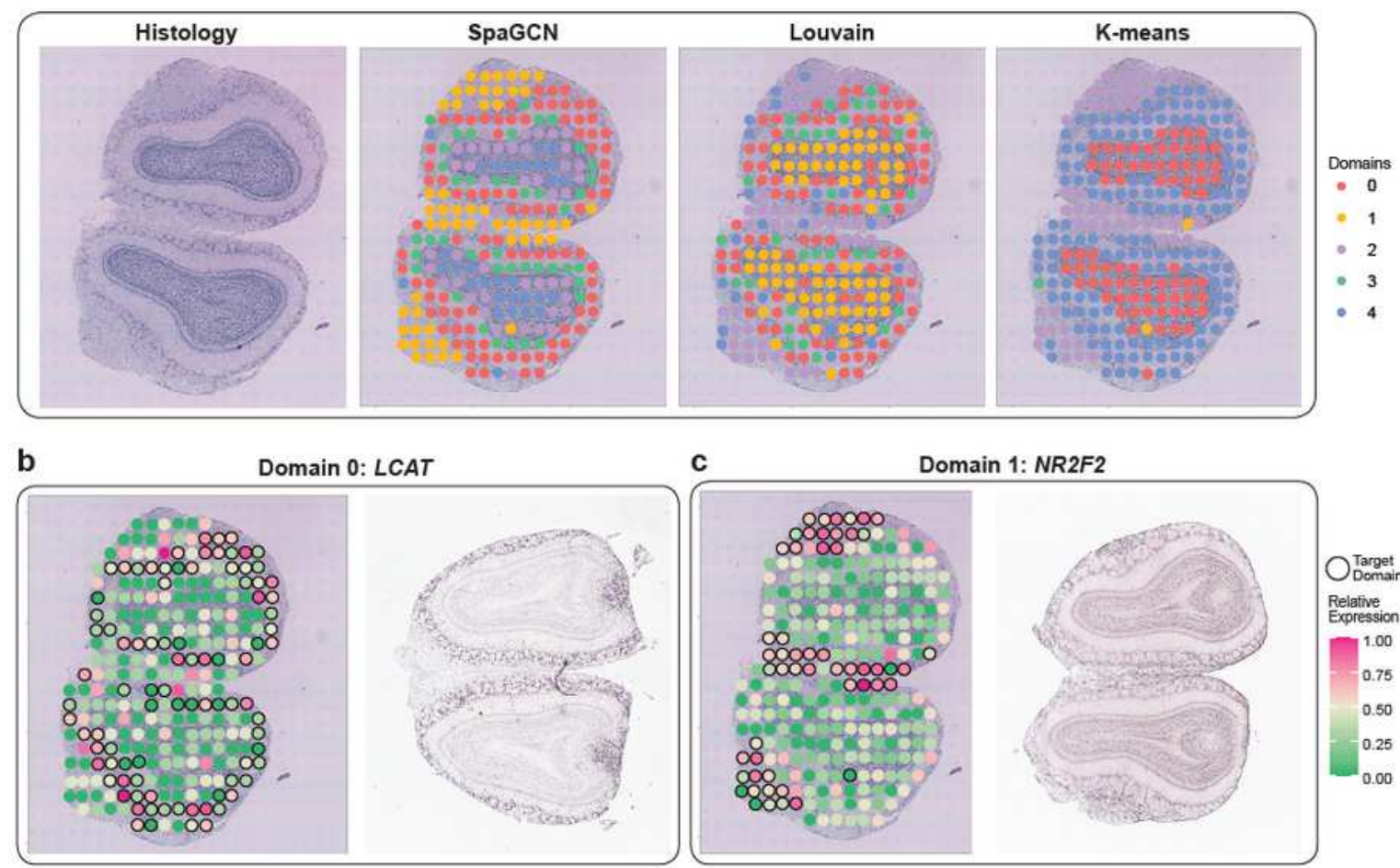

C Domain 1: NR2F2

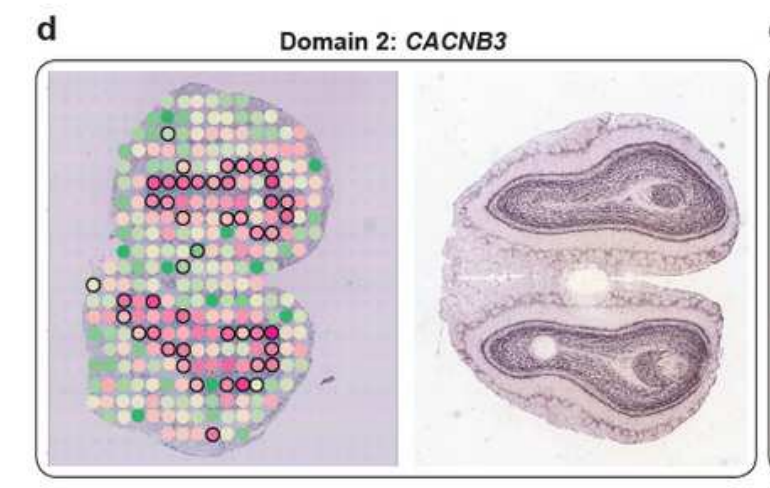

e

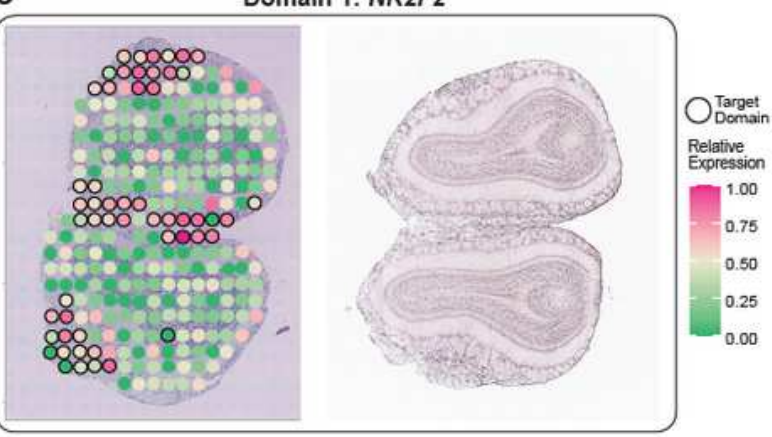

Domain 3: SLC17A7

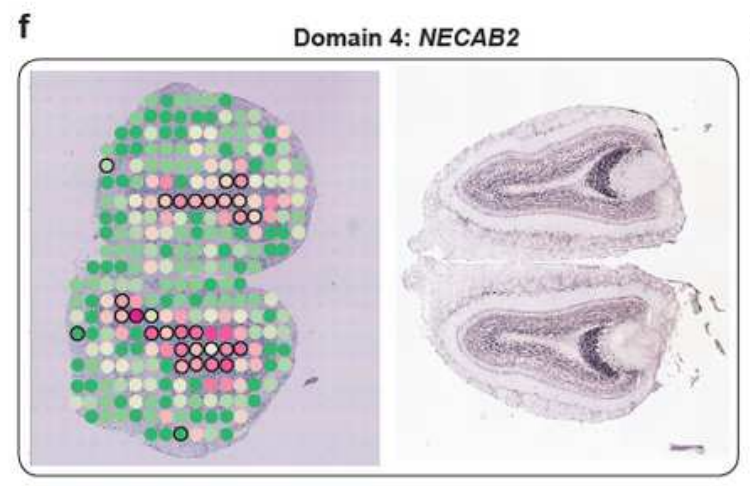

g

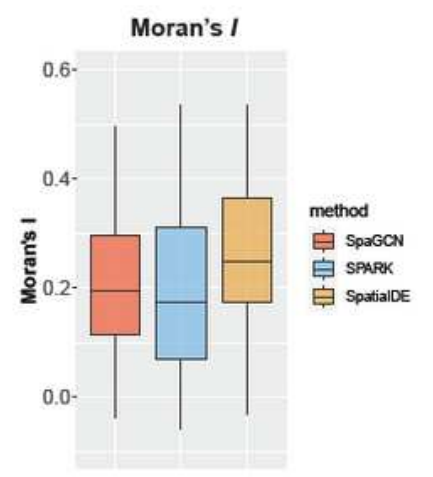

Figure 2 
Spatial domains and SVGs detected in the mouse olfactory bulb dataset. a, Histology image of the tissue section and spatial domains detected by SpaGCN, Louvain's method, and K-means clustering. b-f, Spatial expression patterns of SVGs detected by SpaGCN for domains 0 (LCAT), 1 (NR2F2), 2 (CACNB3), 3 (SLC17A7), and 4 (NECAB2), and the corresponding in situ hybridization of these SVGs obtained from the Allen Brain Atlas. g, Boxplot of Moran's I values for SVGs detected by SpaGCN, SPARK, and SpatialDE.

a
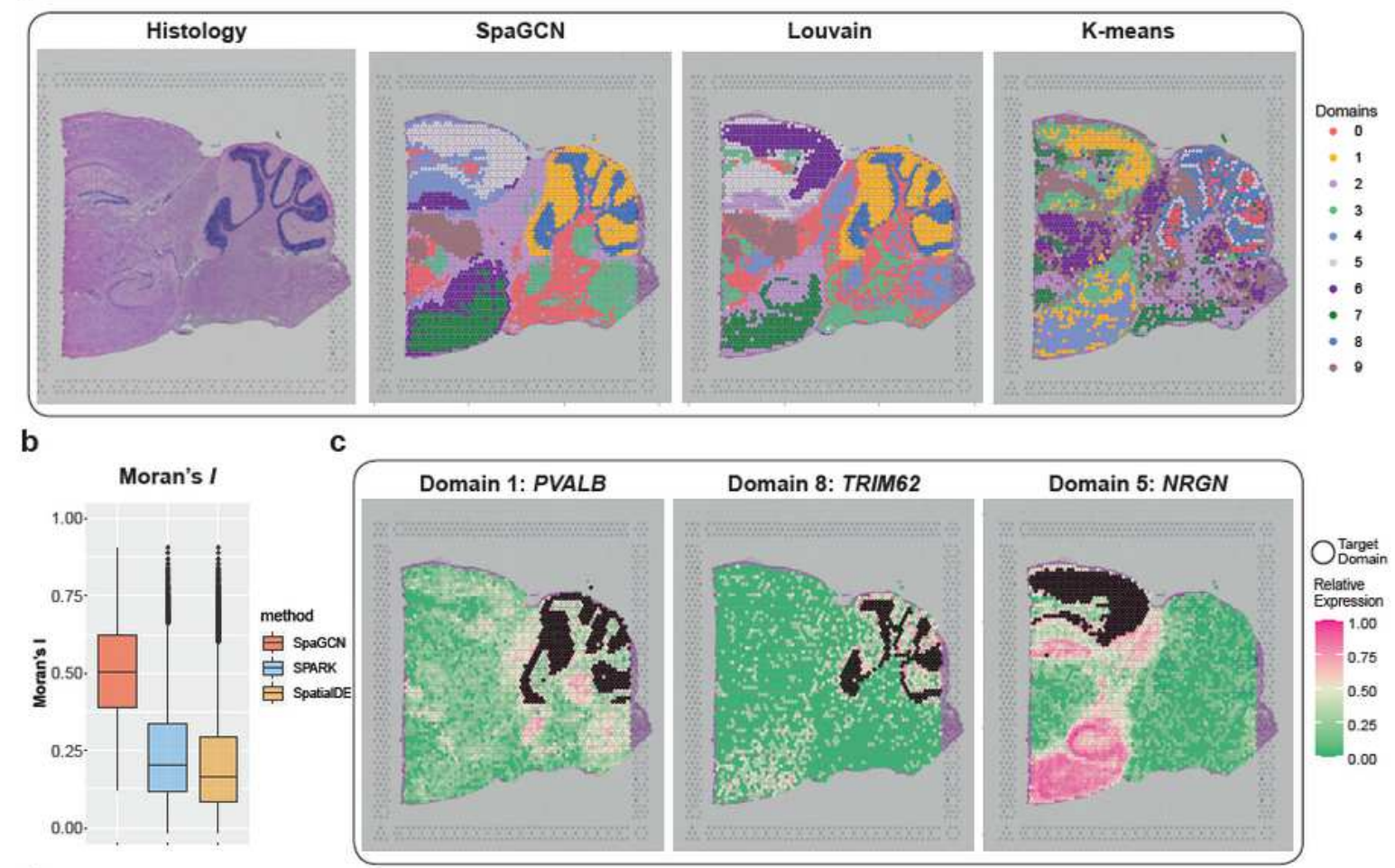

d

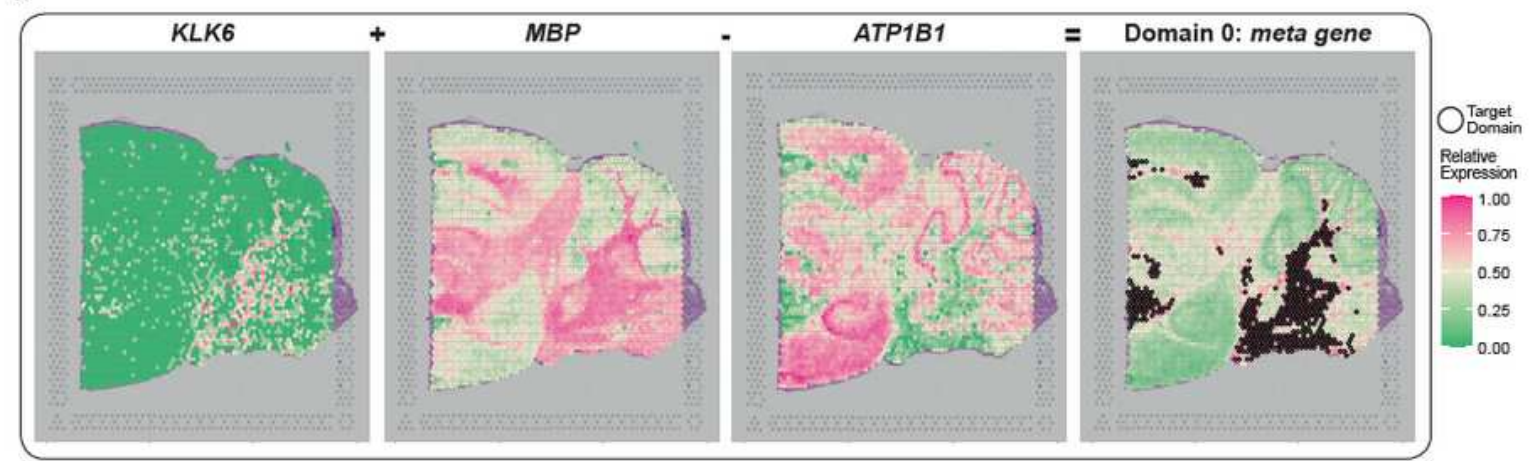

e

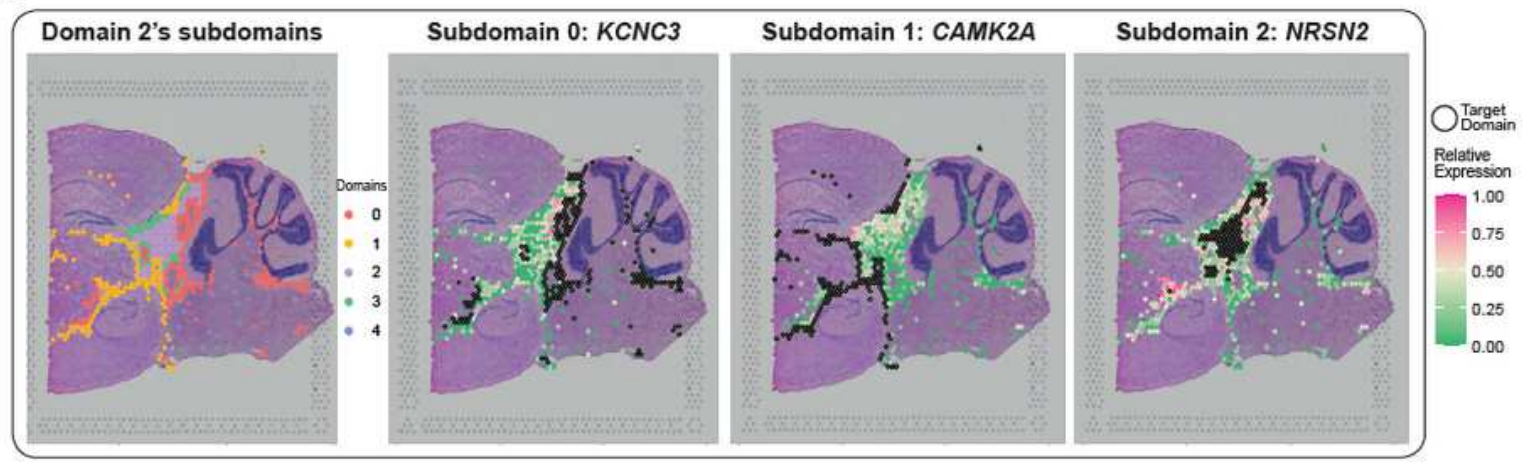

Figure 3 
Spatial domains and SVGs detected in the mouse brain posterior brain dataset. a, Histology image of the tissue section and spatial domains detected by SpaGCN, Louvain's method, and K-means clustering. b, Boxplot of Moran's I values for SVGs detected by SpaGCN, SPARK, and SpatialDE. c, Spatial expression patterns of SVGs detected by SpaGCN for domain 1 (PVALB), 8 (TRIM62), and 5 (NRGN). d, Spatial expression patterns of genes KLK6, MBP, ATP1B1, which form the specific marker meta gene for domain 0 (KLK6 + MBP - ATP1B1). e, Clustering results for 5 sub-domains detected by SpaGCN for domain 2, and the spatial expression patterns of SVGs for sub-domains 0 (KCNC3), 1 (CAMK2A), and 2 (NRSN2).

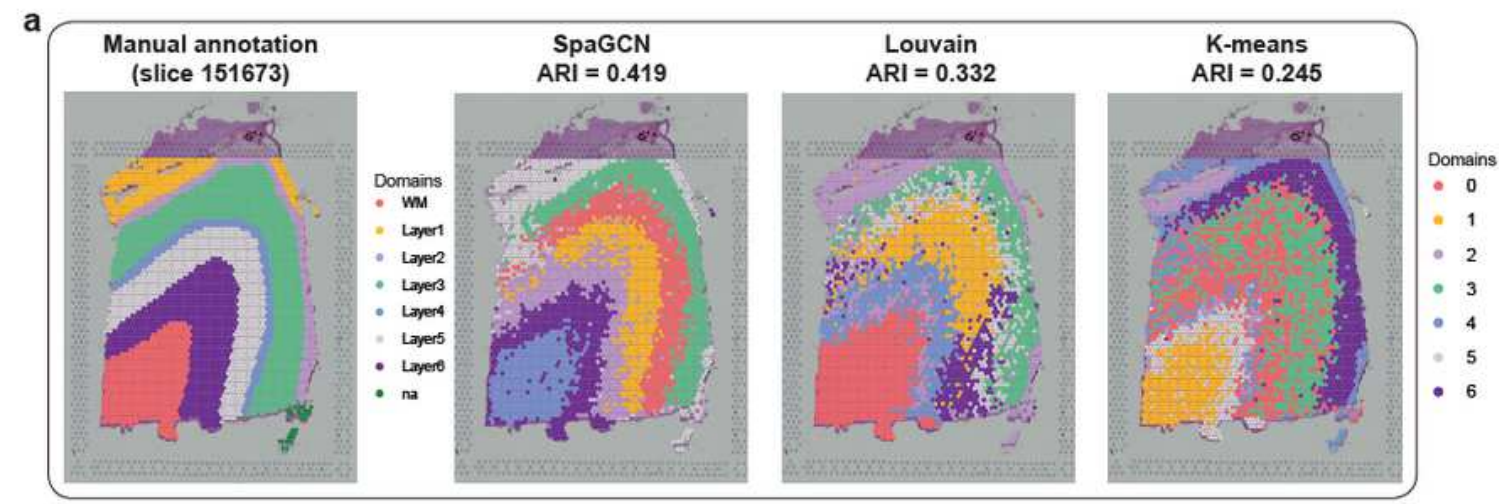

b

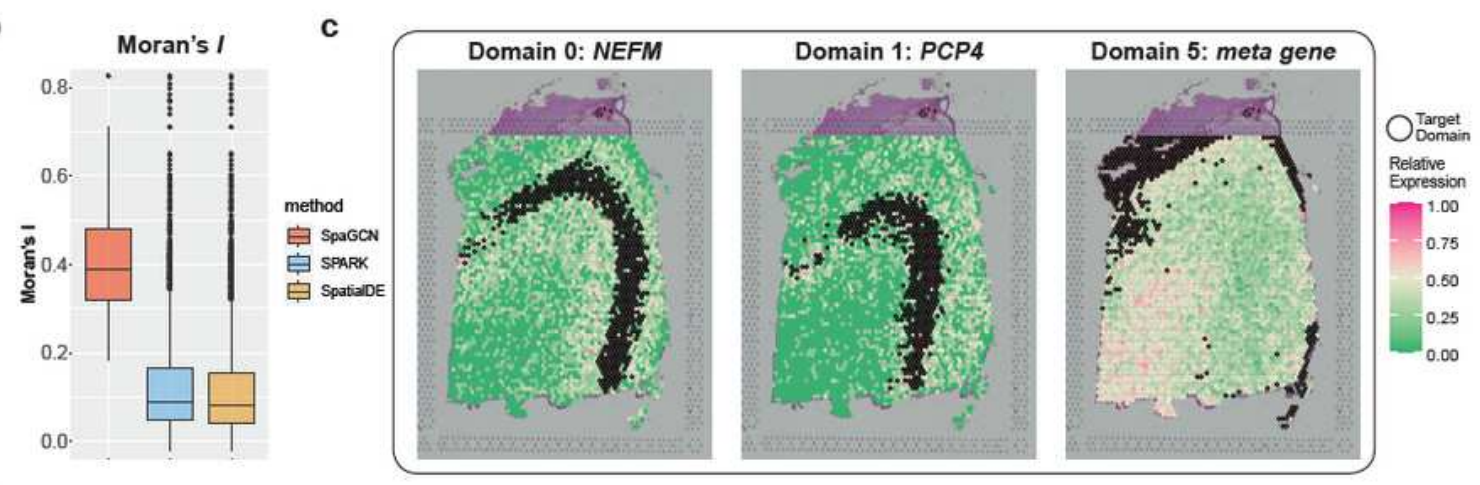

d
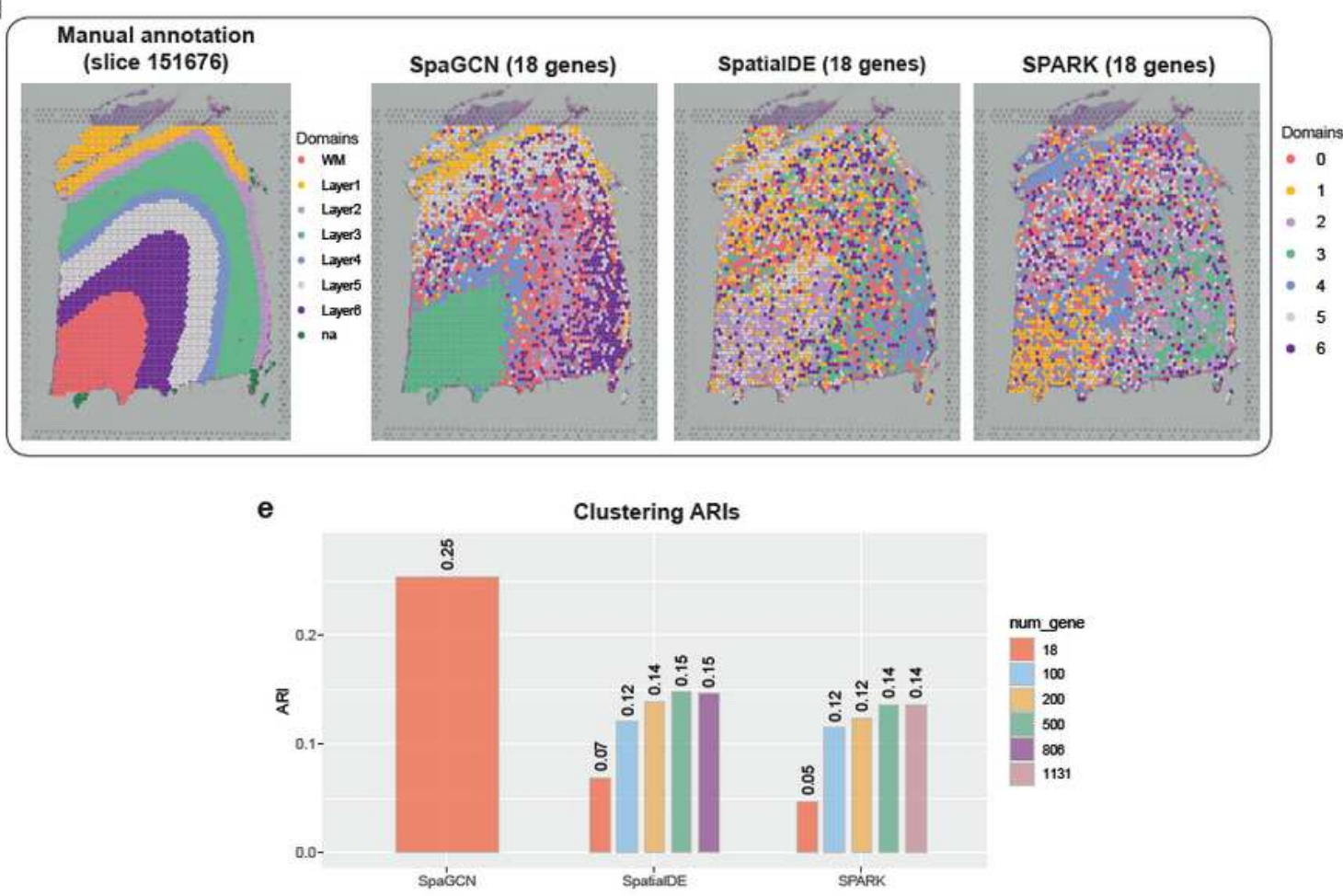


\section{Figure 4}

Spatial domains and SVGs detected in the LIBD human dorsolateral prefrontal cortex dataset. a, Manually annotated layer structure for slice 151673 from the original study20, and spatial domains detected by SpaGCN, Louvain's method, and K-means clustering. b, Boxplot of Moran's I values for SVGs detected by SpaGCN, SPARK, and SpatialDE for slice 151673. c, Spatial expression patterns of SVGs for domain 0 (NEFM) and domains 1 (PCP4), and a meta gene formed by KRT19, MYL9, MBP, GFAP, and SNAP25 for domain 5 (KRT19 + MYL9 - MBP + GFAP - SNAP25). d, Manually annotated layer structure for slice 151676 from the original study20, and K-means clustering results for slice 151676 using 18 genes selected by SpaGCN, SPARK, and SpatialDE. For SpaGCN, we selected the following genes, domain 0 (NEFL, NEFM), domain 1 (PCP4, TMSB10 + PCP4 - KRT19), domain 2 (CCK + KRT17 - MT-ND1, CPLX2 + KRT17 - MT-ND2), domain 3 (CAMK2N1, ENC1), domain 4 (MBP, FTL), domain 5 (KRT19 + MYL9 $\mathrm{MBP}+\mathrm{GFAP}$ - PLP1, KRT8 + MYL9 - MBP + GFAP - PLP1), and domain 6 (GFAP, MBP), resulting in 18 unique genes in total. For SPARK and SpatialDE, the 18 SVGs with the smallest FDR-adjusted $p$-value or q-value were randomly selected. e, ARIs between manually annotated layers and K-means' clustering using SVGs selected by different methods. For SpaGCN, we only used the selected SVGs and meta genes, with 18 genes involved in total while for SPARK and SpatialDE, we used top 18, 100, 200, 500 and all SVGs with the identical smallest FDR-adjusted p-value or q-value. 
a

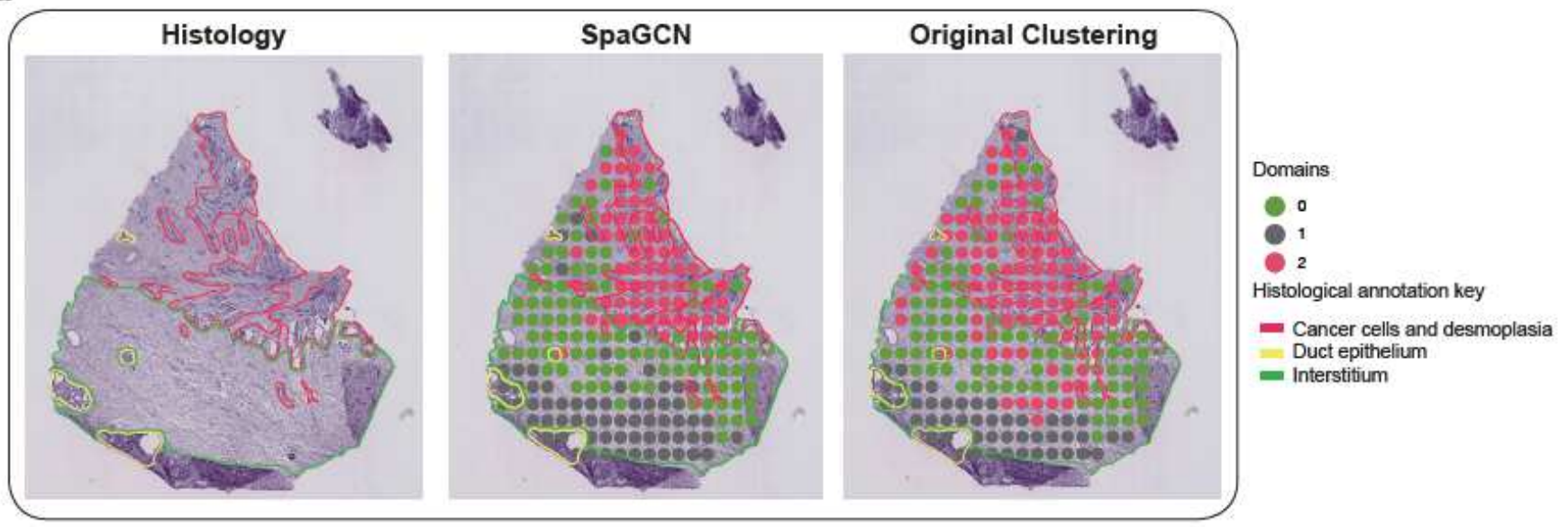

b

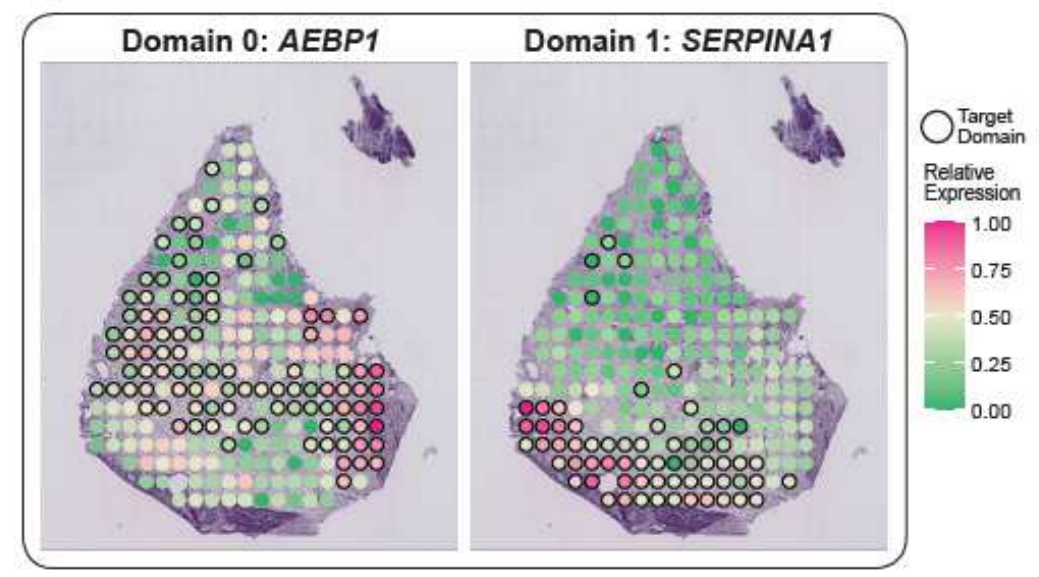

d

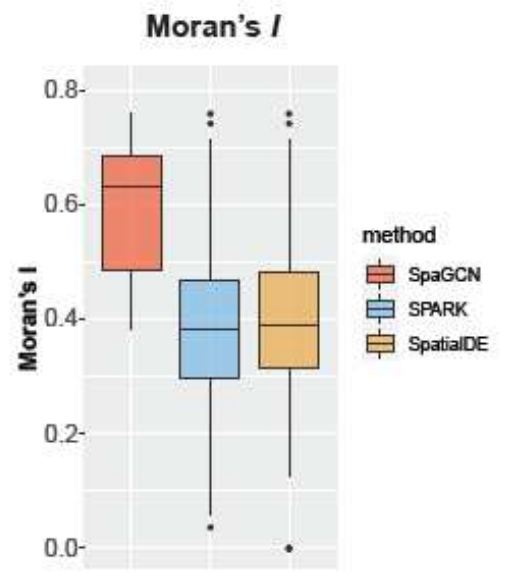

c

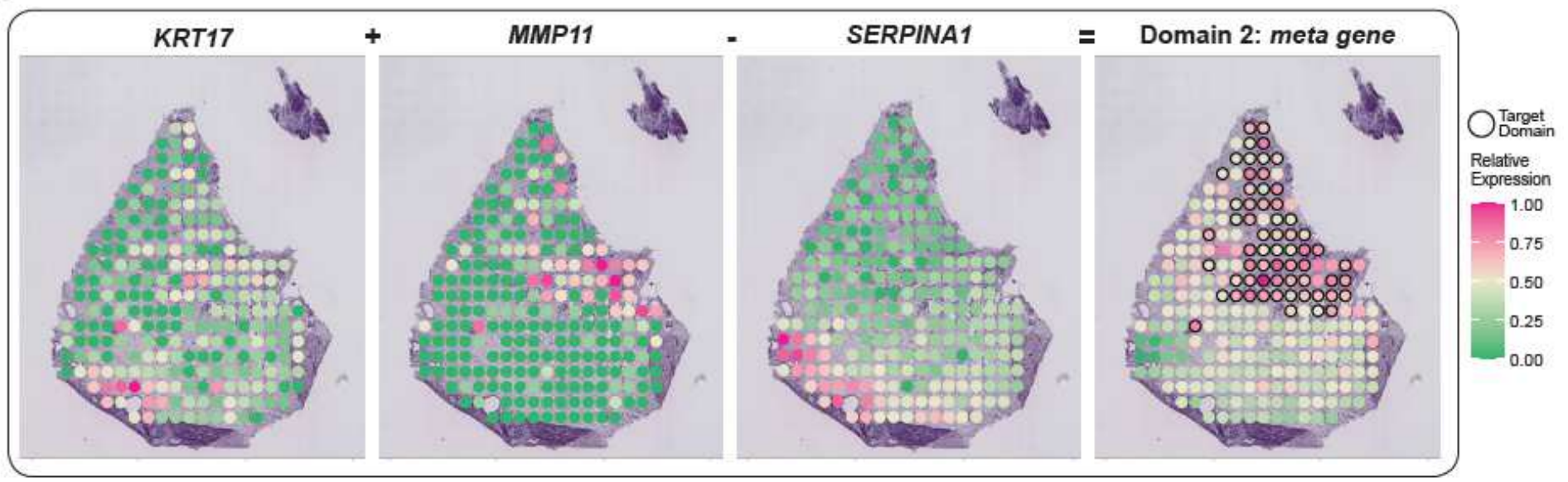

\section{Figure 5}

Spatial domains and SVGs detected in the human primary pancreatic cancer tissue dataset. a, Histology image of the tissue section with manually annotated regions from the original study 5 , spatial domains detected by SpaGCN, and clustering results from the original study. b, Spatial expression pattern of SVGs detected by SpaGCN for domain 0 (AEBP1) and domain 1 (SERPINA1). c, Spatial expression patterns of genes KRT17, MMP11, SERPINA1, which form the specific marker meta gene for domain 2 (KRT17 + 
MMP11 - SERPINA1). d, Boxplot of Moran's I values for SVGs detected by SpaGCN, SPARK, and SpatialDE.

a

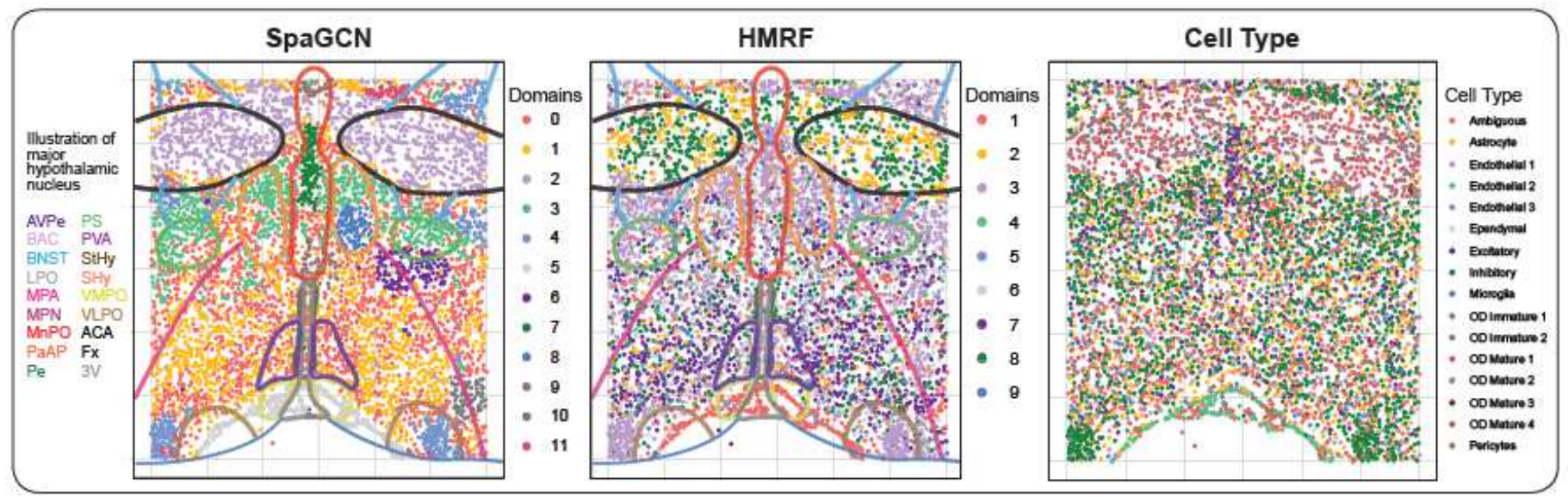

b

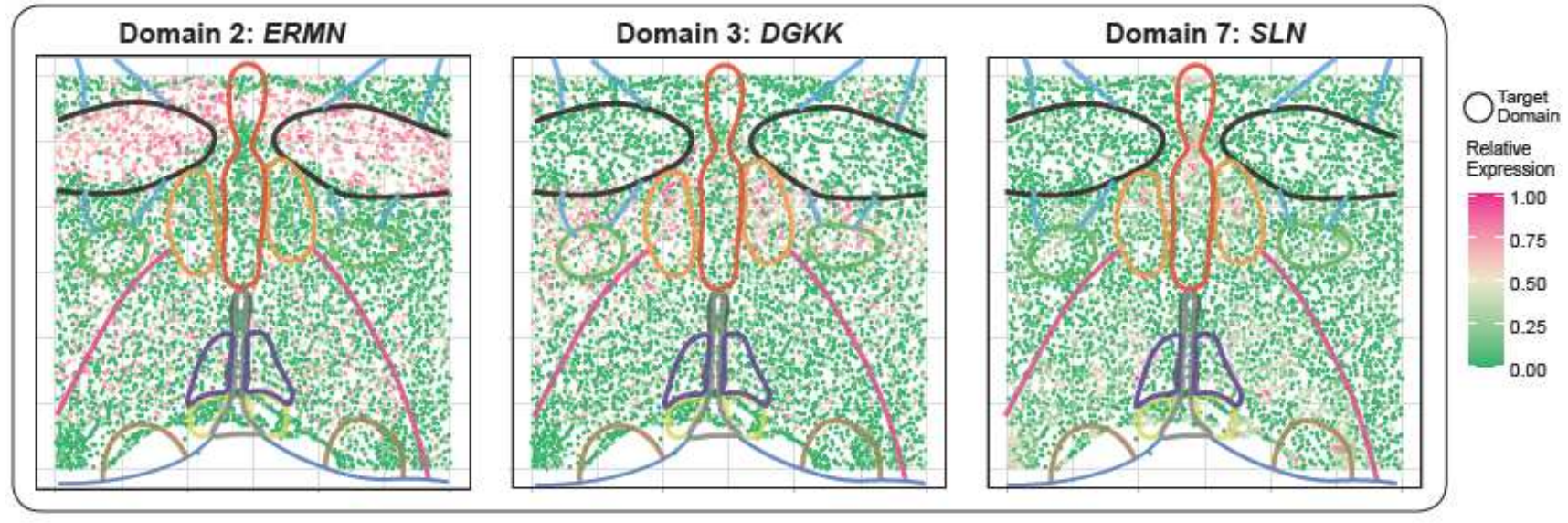

Figure 6

Spatial domains and SVGs detected in the MERFISH mouse brain hypothalamus dataset. a, Spatial domains detected by SpaGCN and the HMRF method overlayed with annotated hypothalamic nuclei from the original study2, and cell type distribution from the original study. $d$, Spatial expression patterns of SVGs detected by SpaGCN for domain 2 (ERMN), domain 3 (DGKK), and domain 7 (SLN).

\section{Supplementary Files}

This is a list of supplementary files associated with this preprint. Click to download.

- SupplementaryInformation11302020.docx 